\title{
Tumor stroma-targeted antibody-drug conjugate triggers localized anticancer drug release
}

\author{
Christopher Szot, ${ }^{1}$ Saurabh Saha, ${ }^{2}$ Xiaoyan M. Zhang, ${ }^{2}$ Zhongyu Zhu, ${ }^{1,3}$ Mary Beth Hilton, ${ }^{1,4}$ Karen Morris, ${ }^{1,4}$ Steven Seaman, ${ }^{1}$ \\ James M. Dunleavey, ${ }^{1}$ Kuo-Sheng Hsu, ${ }^{1}$ Guo-Jun Yu, ${ }^{1}$ Holly Morris, ${ }^{5}$ Deborah A. Swing, ${ }^{5}$ Diana C. Haines, ${ }^{6}$ Yanping Wang, ${ }^{3}$ \\ Jennifer Hwang, ${ }^{3}$ Yang Feng, ${ }^{1,3}$ Dean Welsch, ${ }^{2}$ Gary DeCrescenzo, ${ }^{2}$ Amit Chaudhary, ${ }^{1}$ Enrique Zudaire, ${ }^{1}$ \\ Dimiter S. Dimitrov, ${ }^{3}$ and Brad St. Croix ${ }^{1}$ \\ ${ }^{1}$ Tumor Angiogenesis Unit, Mouse Cancer Cenetics Program (MCGP), National Cancer Institute (NCI), NIH, Frederick, Maryland, USA. ${ }^{2 B i o M e d ~ V a l l e y ~ D i s c o v e r i e s ~ I n c ., ~ K a n s a s ~ C i t y, ~ M i s s o u r i, ~ U S A . ~}{ }^{3}$ Protein \\ Interactions Section, Cancer and Inflammation Program, NCI, NIH, Frederick, Maryland, USA. Basic Research Program, Leidos Biomedical Research Inc., Frederick National Laboratory for Cancer Research \\ (FNLCR), Frederick, Maryland, USA. ${ }^{5}$ Transgenic Core Facility, MCGP, NCI, Frederick, Maryland, USA. ${ }^{6}$ Veterinary Pathology Section, Pathology/Histotechnology Laboratory, Leidos Biomedical Research Inc., \\ FNLCR, Frederick, Maryland, USA.
}

\begin{abstract}
Although nonmalignant stromal cells facilitate tumor growth and can occupy up to $90 \%$ of a solid tumor mass, better strategies to exploit these cells for improved cancer therapy are needed. Here, we describe a potent MMAE-linked antibody-drug conjugate (ADC) targeting tumor endothelial marker 8 (TEM8, also known as ANTXR1), a highly conserved transmembrane receptor broadly overexpressed on cancer-associated fibroblasts, endothelium, and pericytes. Anti-TEM8 ADC elicited potent anticancer activity through an unexpected killing mechanism we term DAaRTS (drug activation and release through stroma), whereby the tumor microenvironment localizes active drug at the tumor site. Following capture of $A D C$ prodrug from the circulation, tumor-associated stromal cells release active MMAE free drug, killing nearby proliferating tumor cells in a target-independent manner. In preclinical studies, ADC treatment was well tolerated and induced regression and often eradication of multiple solid tumor types, blocked metastatic growth, and prolonged overall survival. By exploiting TEM8 $^{+}$tumor stroma for targeted drug activation, these studies reveal a drug delivery strategy with potential to augment therapies against multiple cancer types.
\end{abstract}

\section{Introduction}

Antibody-based drugs are revolutionizing cancer therapy. Because of their exquisite specificity, mAbs are uniquely suited for rationally designed cancer therapies, providing highly selective drugs with reduced toxicities. Although antibodies that block receptor function have become important weapons in the oncologist's arsenal, enhanced potency can be achieved by arming antibodies with small-molecule drugs to create antibody-drug conjugates (ADCs). Importantly, following ADC uptake and cleavage, released cell-permeable warheads can diffuse locally to kill antigen-negative cells, helping alleviate problems associated with target antigen heterogeneity.

Over 100 ADCs are in preclinical development, more than 60 are in clinical development, and 3 are US FDA approved and used clinically for cancer therapy $(1,2)$. Brentuximab vedotin (Adcetris), monomethyl auristatin E-linked (MMAE-linked) anti-CD30 ADC, is

Related Commentary: p. 2750

Authorship note: $C S, S S$, and $X M Z$ contributed equally to this work. Conflict of interest: $Z Z, X M Z, D W, G D, D S D, E Z$, and BSC are inventors of intellectual property related to TEM 8 antibodies and TEM8 antibody-drug conjugates, and BSC and DSD have received research support through a Cooperative Research and Development Agreement (CRADA) with BioMed Valley Discoveries Inc. (BVD).

Submitted: February 12, 2018; Accepted: April 4, 2018.

Reference information: J Clin Invest. 2018;128(7):2927-2943.

https://doi.org/10.1172/JCl120481. used for the treatment of Hodgkin lymphoma; trastuzumab emtansine (T-DM1; Kadcyla), DM1-linked anti-HER2 ADC, is used for the treatment of HER2 ${ }^{+}$breast cancer; and inotuzumab ozogamicin (Besponsa), calicheamicin-linked anti-CD22 ADC, is used for the treatment of adults with relapsed or refractory acute lymphoblastic leukemia (ALL). Most ADCs have been designed to target tumor cells directly and are limited to select groups of antigen-positive patients. Furthermore, heterogeneous target expression on tumor cells can lead to resistance through antigen loss following treatment.

An alternative ADC approach involves targeting the tumor stromal compartment, as malignant tumor growth is driven by dynamic interplay between tumor cells and surrounding stromal cells (3-5). Indeed, in some tumors, stroma can comprise up to $90 \%$ of the total tumor mass $(6,7)$. Because tumor-associated stroma of diverse cancer types share many features, stromal-targeted therapy has potential widespread utility. Furthermore, increased genetic stability of nonmalignant stroma may limit the development of resistance - for example, through target antigen loss (8). Carcinoma-associated fibroblasts (CAFs) are the most prevalent stromal cell type within the tumor and can facilitate tumor growth $(9,10)$. When coinjected with tumor cells, CAFs, but not normal fibroblasts, promote breast and prostate cancer growth $(11,12)$. CAFs are thought to promote tumor growth through immune suppression and secretion of factors that stimulate cancer cell proliferation, invasion, and angiogenesis $(13,14)$. Tumor endothelium also plays a critical role in promoting tumor growth and metas- 
A

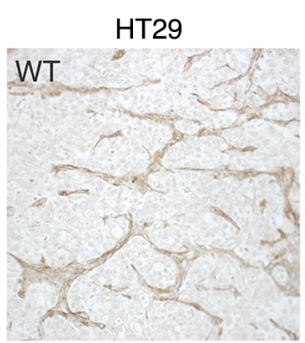

KO
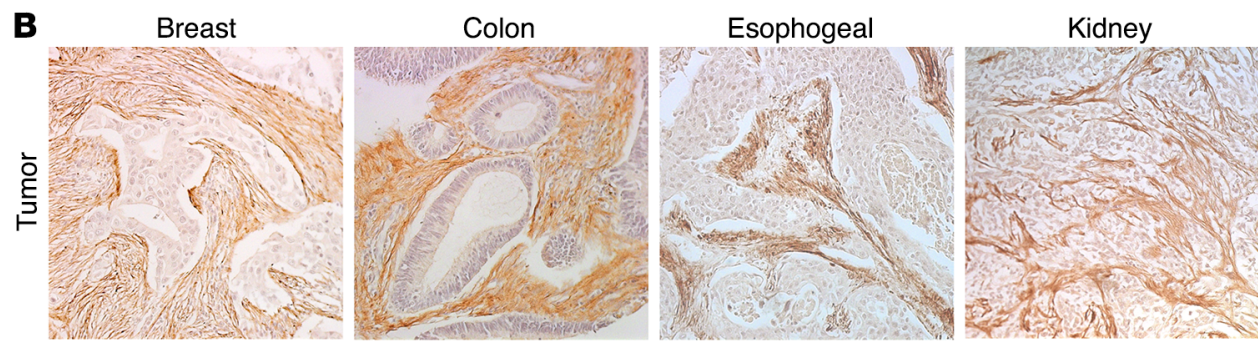

के
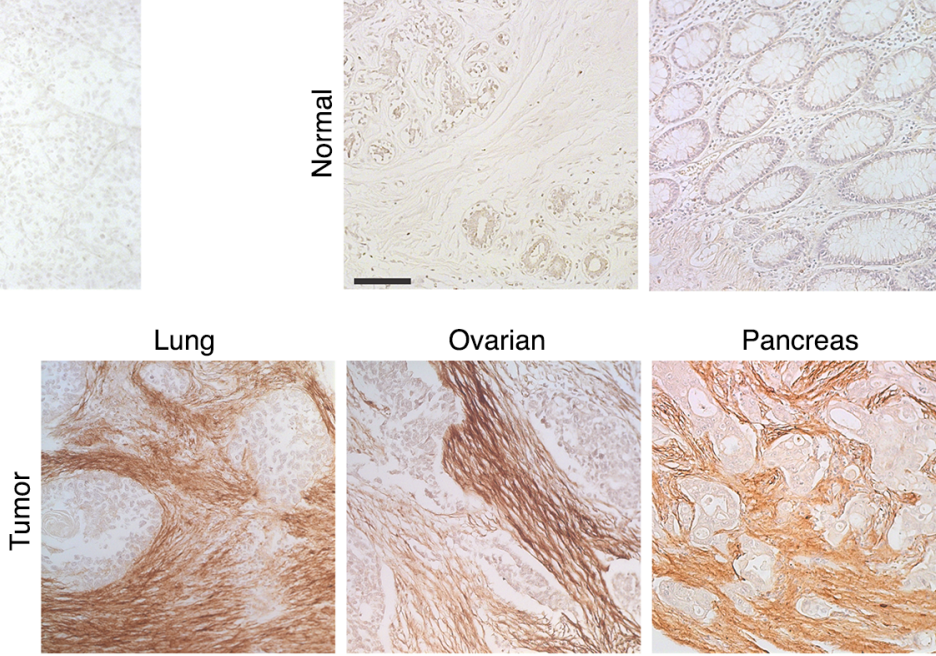

Ovarian

Pancreas

Stomach

Uterus
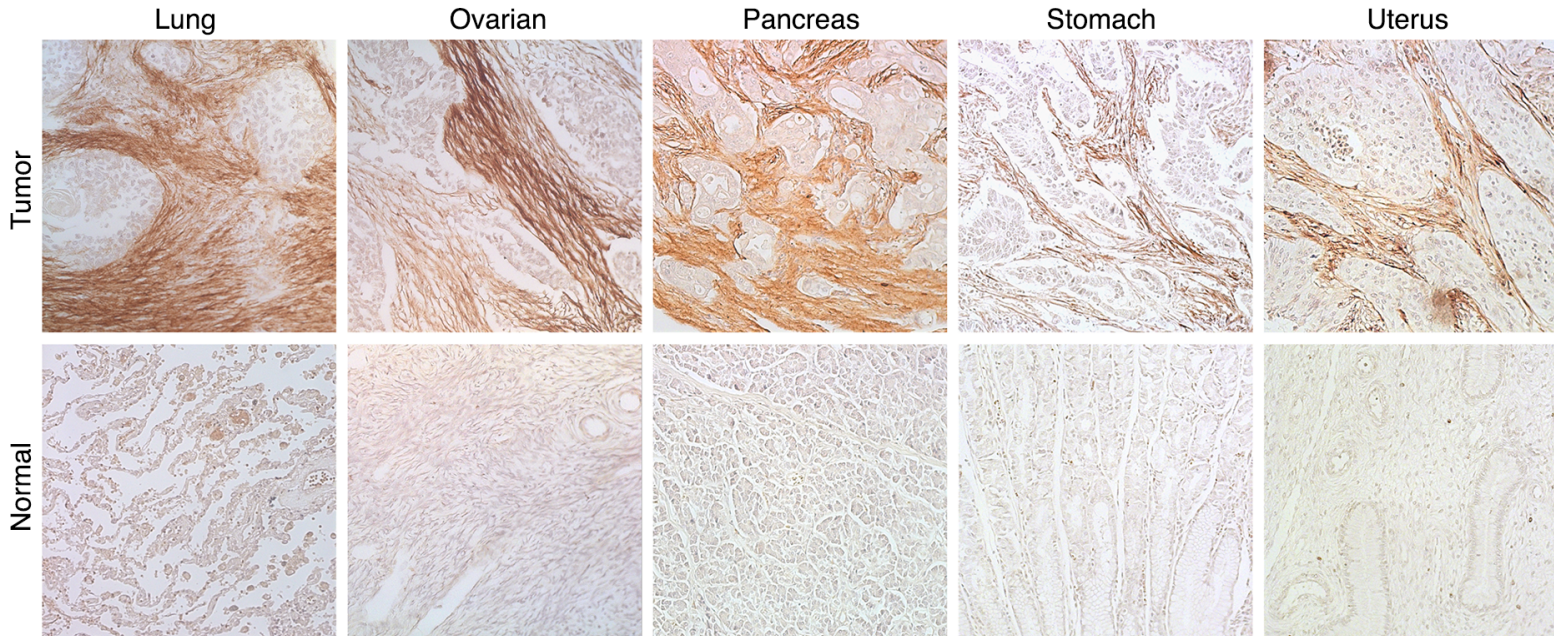

C
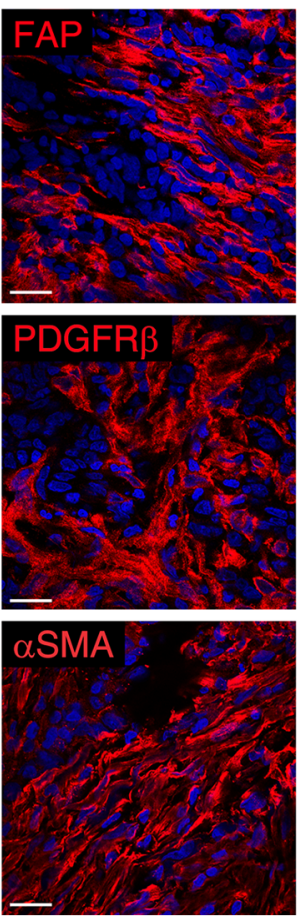
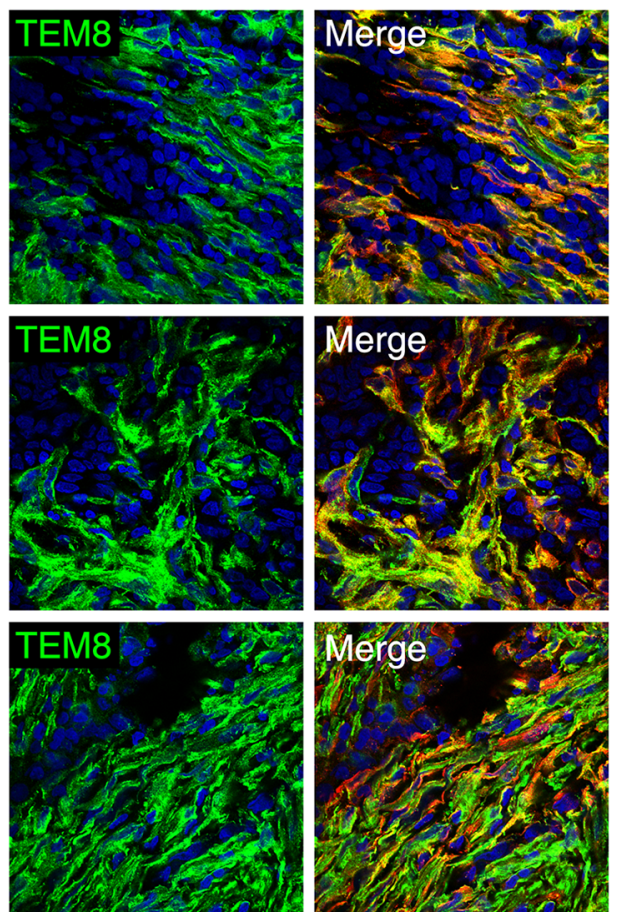
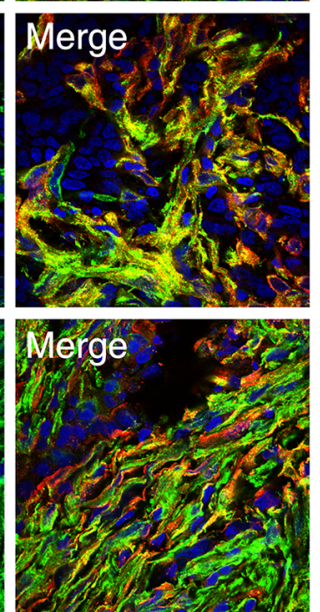

D
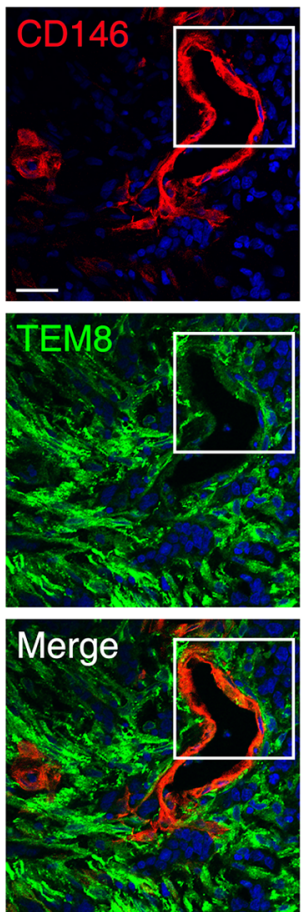
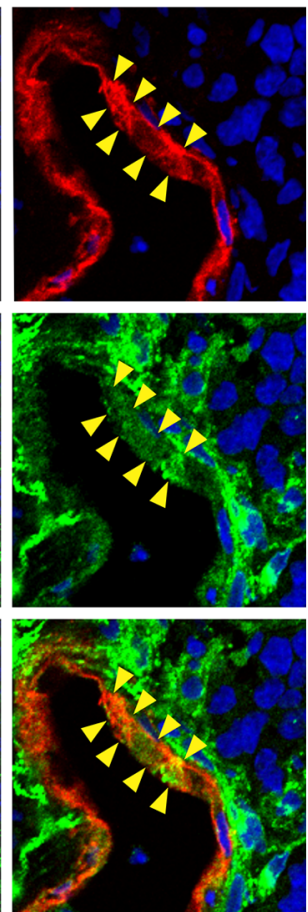
Figure 1. TEM8 is overexpressed in human tumors. (A) Rabbit anti-human TEM 8 mAb was used to stain TEM8 in FFPE sections of HT29 tumors grown in Tem8 WT and Tem8-KO mice. Scale bar: $100 \mu \mathrm{m}$. (B) IHC was used to evaluate TEM8 expression in multiple human tumors or corresponding normal organs. Scale bar: $100 \mu \mathrm{m}$. (C) Co-IF staining of human colorectal tumors for FAP, PDGFR $\beta$, or $\alpha$-SMA (red) and TEM8 (green). Scale bars: 20 $\mu \mathrm{m}$. (D) Co-IF staining of human colorectal tumor for CD146 (red) and TEM8 (green). A double-positive endothelial cell is highlighted (arrowheads). Scale bar: $20 \mu \mathrm{m}$; original magnification, $\times 40$ (insets in D).

tasis, and agents that block angiogenesis through VEGF pathway inhibition are an important component of cancer therapies. However, tumors can adapt by exploiting VEGF-independent pathways of neovascularization, and new approaches to attack tumor endothelium are urgently needed.

While the potential of stromal targeting is widely recognized, innovative approaches have been difficult to implement, largely because of a scarcity of optimal targets with high tumor specificity. Fibroblast activation protein $\alpha$ (FAP), a cell-surface protein overexpressed by most CAFs, was initially considered a stromal cell target but was found to be expressed in normal tissues, which has dampened enthusiasm for FAP targeting $(9,15-17)$. Fortunately, advances in genomics and proteomics have revealed antigens highly expressed on tumor-associated stroma, which may have the specificity needed to develop a stromal cell-directed ADC (18-20).

Tumor endothelial marker 8 (TEM8, also known as ANTXR1) is a highly conserved 80 - to $85-\mathrm{kDa}$ single-pass, cell-surface transmembrane glycoprotein originally identified on the basis of its upregulation in human tumor endothelium $(19,21)$. TEM8 was also found to be widely expressed on tumor-associated perivascular stromal cells, although nonendothelial TEM8 ${ }^{+}$stromal cells have not been fully characterized (22-24). TEM8 upregulation during pathological (tumor) angiogenesis, but not normal angiogenesis, suggests that TEM8 targeting may have minimal offtarget toxicities (25). While TEM8 function in normal physiology remains unclear, TEM8 can bind collagen types I and VI and aid in cell spreading and migration on collagen I in vitro (21, 26-28). Furthermore, while wound healing was unaffected inTem 8 WT versus KO mice, the growth of breast, colon, lung, and melanoma tumors was significantly delayed $(23,29)$. In preclinical studies, treatment with naked TEM8 antibodies slowed tumor growth and prolonged survival through a mechanism that may involve function-blocking activity or antibody-dependent cellular cytotoxicity (23). However, no tumor regressions in response to the monotherapy were observed. Here, we set out to determine whether TEM8 could provide a useful target for the development of a more potent stromal cell-directed ADC. We describe the preclinical development of m825-MMAE, a TEM8 ADC with potent tumor-regressing activity against multiple cancer types and an unexpected tumor-killing mechanism that depends on tumor-associated stroma.

\section{Results}

TEM8 is broadly expressed in human tumor-associated stroma. Previous studies reported high TEM8 mRNA and protein expression levels throughout the stroma of a small number of colon, lung, esophageal, bladder, and breast cancers $(21,23,24,30)$. To further explore TEM8 expression patterns, we performed IHC on 172 normal human and 563 tumor formalin-fixed, paraffin-embedded (FFPE) tissue sections. For this, we generated a rabbit mAb (clone 37) that reacted with the extracellular domain (ECD) of both mouse and human TEM8. Immunoblotting a TEM8-GST deletion series followed by peptide mapping revealed that the antibody recognized a 15 -amino acid $\mathrm{N}$-terminal region that is $100 \%$ conserved between mouse and human TEM8 but differs by 1 amino acid with rabbit TEM8 (Supplemental Figure 1, A-D; supplemental material available online with this article; https://doi.org/10.1172/ JCI120481DS1). IHC on TEM8- HT29 tumors grown in Tem8 WT and Tem8-KO mice verified the specificity for TEM 8 in tumorassociated stroma (Figure 1A). TEM8 IHC revealed wide stromal expression in most tumors analyzed ( $71 \%$ overall), but TEM 8 was undetectable in almost all corresponding adjacent normal tissues (Figure 1B and Supplemental Figure 2A). One exception was normal kidney, in which we observed weak positive glomeruli staining in 2 of 22 samples. Importantly, when frozen sections from 17 primary breast cancer tumors and metastatic colon tumors were analyzed by immunofluorescence (IF) staining, all samples showed high stromal TEM8 with no signal detected in normal adjacent tissue (Supplemental Figure 2, A and B), suggesting that TEM8 antigen may have been sensitive to variations in the FFPE fixation conditions used and that TEM8 positivity in FFPE tumor tissues was likely an underestimate, as noted for other antibodies $(31,32)$.

Next, we evaluated TEM8 expression in 44 different normal adult mouse organs or tissues taken from Tem $8 \mathrm{WT}$ and Tem 8 -KO mice. TEM8 expression was undetectable in all normal tissues examined, except lung and brain. In lung, faint positive staining appeared throughout, whereas in brain, we detected weak TEM8 expression only in choroid plexus epithelium (Supplemental Figure 2C). These experiments indicate that TEM8 is most highly expressed in tumor-associated stroma and represents a potential target for ADC development.

TEM8 is expressed in tumor-associated fibroblasts, pericytes, and endothelium. While TEM8 was originally identified in tumor endothelial cells, subsequent studies revealed widespread TEM8 expression throughout the tumor stroma $(21,23)$. To determine which stromal cell types express TEM8, we performed co-IF staining on human colorectal tumors. TEM 8 colocalized strongly with CAF markers, including FAP, $\alpha$-smooth muscle actin ( $\alpha$-SMA), and PDGFR $\beta$ (Figure 1C). While TEM8 was expressed in stromal cells throughout the tumor (Supplemental Figure 3), fibroblast expression was heterogeneous, with high levels detectable in some, but not all, $\mathrm{FAP}^{+}, \alpha-\mathrm{SMA}^{+}$, and $\mathrm{PDGFR} \beta^{+}$stromal cells. Although fibroblasts were the most prominent $\mathrm{TEM}^{+}$stromal cell type, in some tumors TEM8 was detectable in CD146 ${ }^{+}$endothelials cells (ECs) and desmin ${ }^{+}$pericytes (Figure 1D and Supplemental Figure 4) as previously described (33).

Development of a fully human, high-affinity TEM8 ADC. Given the widespread TEM8 overexpression in tumor stroma, we sought to develop an anti-TEM8 ADC. We screened a diverse human scFv yeast antibody display library to identify a fully human $\mathrm{mAb}$ for ADC development. Importantly, in vitro antibody display avoids tolerance mechanisms, allowing identification of antibodies against highly conserved epitopes. One antibody, m825, was isolated after repeated screening with mouse and human TEM8 ECD and selected for ADC development, because it showed high affini- 
A
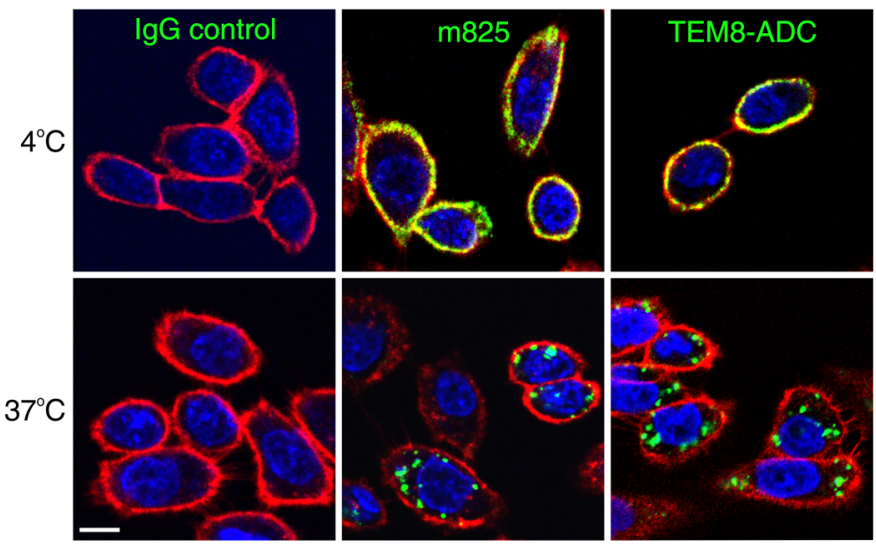

B

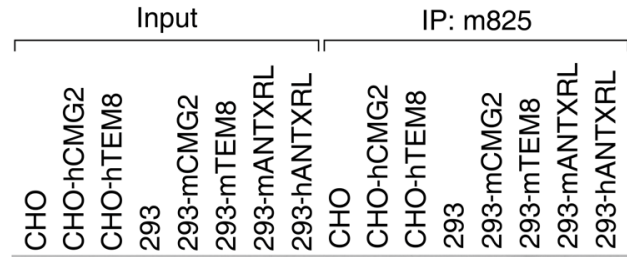

IB Ab:

$\alpha$ TEM8

(c37)

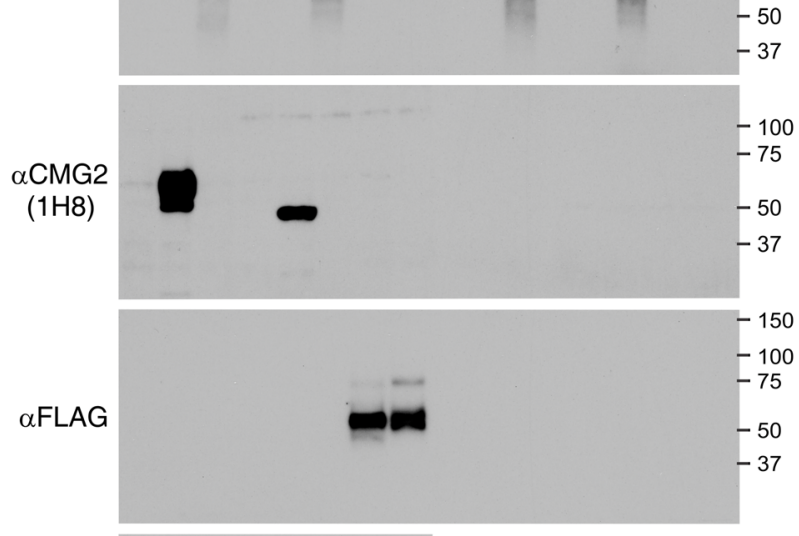

aGAPDH
C

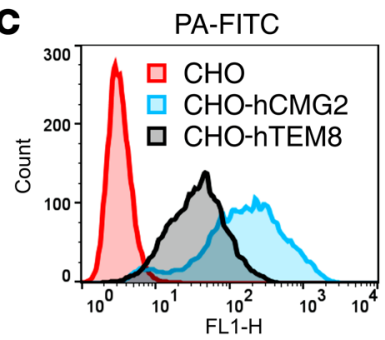

PA-FITC

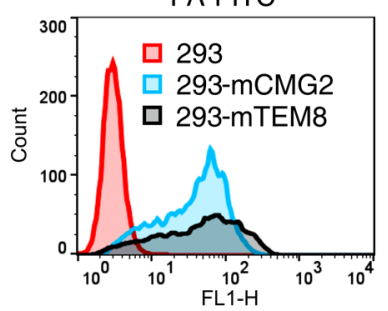

Anti-FLAG
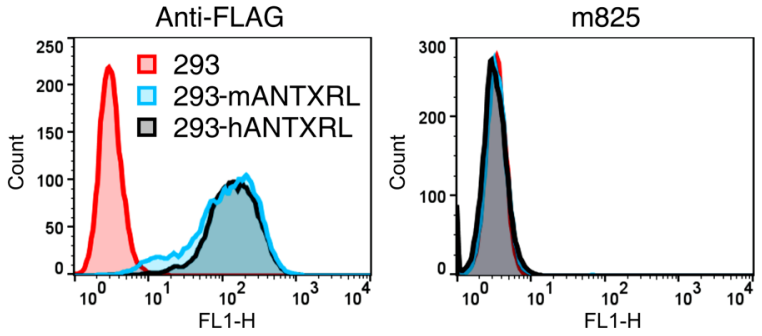

D

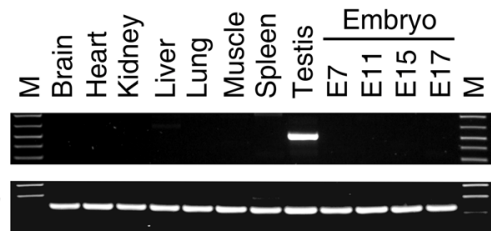

E

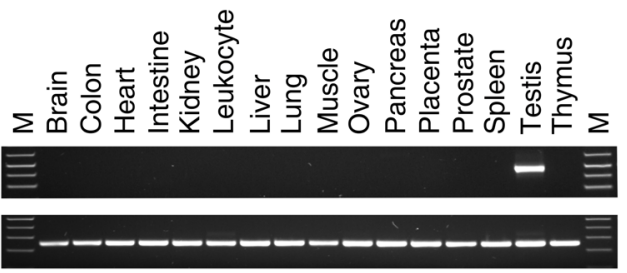

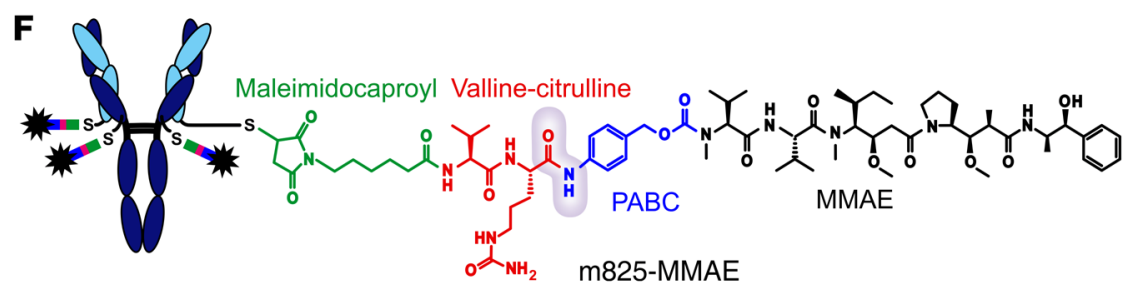

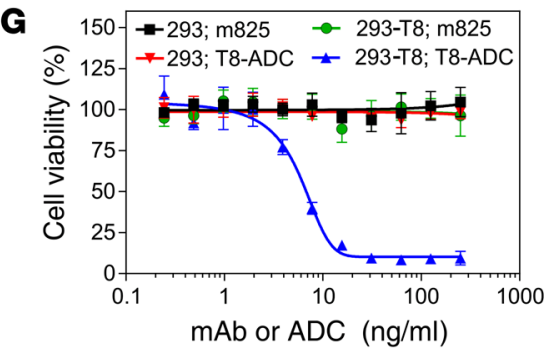


Figure 2. $\mathbf{m} 825$ specifically binds mouse and human TEM8. (A) Uptake of $\mathrm{m} 825$ or m825-MMAE (TEM8-ADC, green) was evaluated in CHO-TEM8 cells after shifting cells from $4^{\circ} \mathrm{C}$ to $37^{\circ} \mathrm{C}$ for 60 minutes. Cell membranes were counterstained with CellMask Orange. Scale bar: $10 \mu \mathrm{m}$. (B) m825 IP of proteins from CHO cells stably expressing human CMC2 or TEM8, or 293 cells stably expressing mCMC2, mTEM8, mANTXRL, or hANTXRL. Immunoprecipitated proteins were immunoblotted (IB) with anti-TEM8 mAb ( $\alpha$ TEM8) (rabbit c37), anti-CMG2 mAb (clone 1H8), and anti-FLAG mAb. GAPDH was used as a loading control. (C) Flow cytometry was used to evaluate m825-MMAE reactivity in CHO cells stably expressing hCMC2 or hTEM8, or 293 cells expressing mCMG2, mTEM8, mANTXRL, or hANTXRL. As a positive control, PA-FITC was used to bind both CMC2 and TEM8, and anti-FLAG antibodies were used to detect mouse and human FLAG-tagged ANTXRL proteins. (D) RT-PCR was used to evaluate mouse Antxrl mRNA expression in various adult organs and E7, E11, E15, and E17 whole embryos. (E) RT-PCR was used to evaluate human ANTXRL mRNA expression in various adult organs. (F) Chemical structure of m825-MMAE linker and warhead. The maleimidocaproyl attachment group (green), p-aminobenzylcarbamate (PABC) spacer (blue), and the cathepsin B-cleavable valinecitrulline dipeptide (red) are indicated. The gray cloud highlights the amide group susceptible to cleavage by carboxylesterase 1C in mouse serum. (C) Cell viability assays were used to measure the activity of m825 naked $A b$ or m825-MMAE (T8-ADC) against 293 or 293 cells overexpressing human TEM8 (293-T8). Data represent the mean \pm SD. M, molecular weight marker.

ty for TEM8 $\left(K_{D}: 59 \mathrm{pM}\right)$, was specifically internalized into TEM $8^{+}$ cells, and showed favorable characteristics for antibody production, including high yields and stability (Figure 2A and Table 1).

TEM8 shares $54 \%$ amino acid ECD identity with capillary morphogenesis protein-2 (CMG2, also known as ANTXR2), the primary anthrax toxin receptor and second ANTXR family member identified following TEM8. When we evaluated m825 for specificity in IP and performed flow cytometric studies using mouse and human TEM8- or CMG2-expressing cells, we observed only murine TEM8 (mTEM8) and human TEM8 (hTEM8) binding (Figure 2, B and C). Upon searching nucleotide databases for other possible TEM8 homologs, we identified a previously uncharacterized third ANTXR family member in cDNA samples from testis. We sequenced human and mouse cDNA from testis and identified full-length ORFs, called ANTXR-like (ANTXRL), encoding putative transmembrane receptors (GenBank accession numbers KY947541 and KY947542). The ECD of ANTXRL, which contains a single vWA domain similar to that of the other ANTXR family members, shares $45 \%$ amino acid identity with TEM8 and $41 \%$ amino acid identity with CMG2. PCR screening of mouse and human cDNA panels derived from various adult and embryonic tissues revealed expression only in testis (Figure 2, D and E). Overexpression of FLAG-tagged mouse or human ANTXRL in HEK293 cells (referred to hereafter as 293 cells) revealed a protein of approximately 55 to $60 \mathrm{kDa}$ (Figure 2B). Flow cytometric staining verified that both mouse and human ANTXRL proteins, like TEM8 and CMG2, were expressed on the cell surface (Figure 2C). Importantly, flow cytometry and IP with m825 revealed no detectable cross-reactivity with mouse or human ANTXRL, verifying the specificity for TEM8 (Figure 2, B and C).

To construct the TEM8 ADC, m825 was linked to MMAE, a potent microtubule-disrupting synthetic analog of the murine natural product dolastatin 10 (34), via a cathepsin B-cleavable valine-citrulline dipeptide linker (Figure $2 \mathrm{~F}$ ), the same druglinker design used for clinically approved brentuximab vedotin.
The cathepsin B site was incorporated into the linker to facilitate MMAE release from the antibody upon internalization into lysosomes. m825-MMAE contained an average of 4 drug molecules per $\mathrm{mAb}$, the optimal drug-to-antibody ratio found in previous studies (35). Binding and internalization of $\mathrm{m} 825$ and $\mathrm{m} 825$-MMAE were indistinguishable by ELISA and cell uptake assays (Figure 2A and Supplemental Figure 5A). Although m825-MMAE was extremely stable in human serum, in mouse serum, analysis by liquid chromatography tandem mass spectrometry (LC-MS/MS) revealed the gradual release of up to $24 \%$ free MMAE by 3 weeks (Supplemental Figure $5 \mathrm{~B})$, in agreement with previous findings $(36,37)$. The partial instability of MMAE-ADCs in mouse serum has been attributed to low-level cleavage of dipeptide linkers by carboxylesterase 1C, an enzyme found in mouse but not human serum (38).

We monitored in vitro cell viability with m825-MMAE in 293 parent cells and 293/TEM8 cells stably transfected with TEM8. We found that 293/TEM8 cells were effectively killed by the ADC, while parental 293 cells were unaffected (Figure $2 \mathrm{G}$ ). As expected, membrane-permeable MMAE free drug displayed indiscriminate cytotoxicity against 293 and 293/TEM8 cells, while parental mAb m825 had no cytotoxic activity (Figure 2G). Thus, m825-MMAE selectively kills TEM8-expressing cells.

m825-MMAE regresses s.c. and orthotopic tumor growth and prolongs survival. To examine m825-MMAE efficacy in vivo, immunodeficient athymic nu/nu mice were challenged with human colon (HCT-116, HT29, and DLD-1), breast (MDA-MB-231), lung (DMS273 and HOP92), ovarian (OVCAR3), and pancreatic (HPAC) tumor xenografts (Figure 3, A-H). Immunocompetent C57BL6 mice were also challenged with MC38 colon and B16 melanoma tumors (Figure 3I and Supplemental Figure 6). Treatments began once tumors reached an average size of $100 \mathrm{~mm}^{3}$. While TEM8ADC elicited potent dose-dependent antitumor activity against HCT-116, MDA-MB-231 and DMS-273, from 3 to $30 \mathrm{mg} / \mathrm{kg}$, equivalent parent $\mathrm{m} 825$ antibody doses only evoked modest reductions in tumor growth (Figure 3, A-C). Although a single $10 \mathrm{mg} / \mathrm{kg}$ dose of TEM8-ADC could arrest orthotopic MDA-MB-231 breast tumor growth for 3 weeks (Supplemental Figure 7), dosing with $10 \mathrm{mg} / \mathrm{kg}$ twice per week for 3 weeks resulted in striking tumor regressions (Figure 3B). We observed tumor regressions in most tumor types (Figure $3, A_{-}-$), with $10 \%$ to $80 \%$ of the mice being tumor free following treatment, depending on the model. Intravenous versus intraperitoneal ADC administration in the DMS-273 model showed no difference in efficacy (Figure 3C). We found that m825-MMAE was also highly effective against lung (COS-G) and breast (CLO-G) cancer patient-derived xenograft (PDX) models, inducing a complete regression of many tumors, even when treatment was initiated after tumors reached a relatively large size $\left(1,000 \mathrm{~mm}^{3}\right)$ (Figure 3, J and K, and Supplemental Figure 8). Importantly, TEM8-ADC

Table 1. Affinity of monovalent $\mathbf{m} 825$ Fab for TEM8 protein

$\begin{array}{lccc}\text { Target antigen } & \mathbf{K}_{\text {on }}\left(\mathbf{M}^{-1} \mathbf{s}^{-1}\right) & \mathbf{K}_{\text {off }}\left(\mathbf{s}^{-1}\right) & \mathbf{K}_{\mathbf{D}}(\mathbf{M}) \\ \text { Mouse TEM8 } & 1.7 \times 10^{6} & 8.9 \times 10^{-5} & 5.3 \times 10^{-11} \\ \text { Human TEM8 } & 3.1 \times 10^{6} & 1.8 \times 10^{-4} & 5.9 \times 10^{-11}\end{array}$



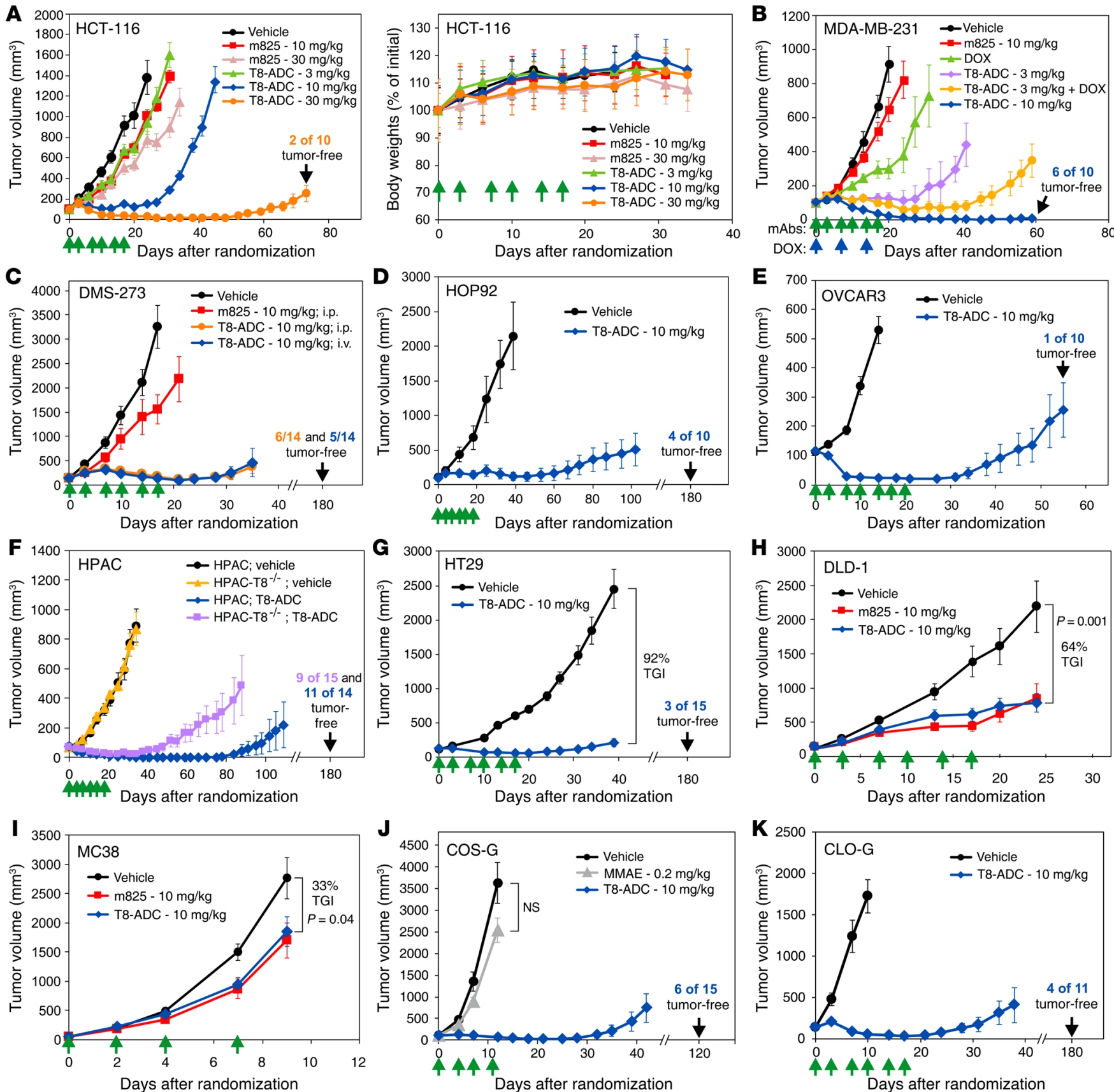

Figure 3. m825-MMAE elicits potent antitumor activity against various tumors, without evidence of toxicities. (A-I) Growth of s.c. human colon HCT-116 (A, left), lung DMS-273 (C), HOP92 (D), ovarian OVCAR3 (E), pancreatic HPAC (F), colon HT29 (C), DLD-1 (H), or mouse MC38 (I), and orthotopic human breast MDA-MB-231 (B) tumors. (A, right) Body weights of the mice in the HCT-116 tumor experiment shown in $\mathbf{A}$ (left). (J and $\mathbf{K}$ ) Growth of human s.c. lung COS- $\mathrm{C}$ (J) or orthotopic breast CLO-G (K) PDX tumors. Treatments with vehicle, MMAE, m825, or m825-MMAE (T8-ADC) were initiated when tumors reached an average size of approximately $100 \mathrm{~mm}^{3}$ and were administered on the indicated days (green arrows). Blue arrows indicate doxorubicin (DOX) treatment days. $n \geq 10$ / group (A-J). Data represent the mean \pm SEM. The $P$ values in $\mathbf{H}$ and I were determined by Student's $t$ test.

was much more potent than a nontargeted MMAE-linked control ADC (Supplemental Figure 9), and treatment with $0.2 \mathrm{mg} / \mathrm{kg}$ free MMAE, equivalent to free drug load on m825-MMAE at $10 \mathrm{mg} / \mathrm{kg}$, had no significant impact on tumor growth (Figure 3J).

Although TEM8 expression in vivo is highest in tumor-associated stroma, low levels of TEM8 were detected by flow cytometry on some cancer cell lines (Supplemental Figure 10). To determine whether TEM8 expression levels in tumor cells were sufficient to affect ADC efficacy, TEM8 was disrupted in two TEM8 ${ }^{+}$cancer cell lines, DMS-273 (lung) and HPAC (pancreatic), using CRISPR-Cas9 (Supplemental Figure 10). We found that disruption of TEM8 did not affect the tumor growth rate in the vehicle control groups (Figure $3 \mathrm{~F}$ and Supplemental Figure 11). However, TEM $8^{+}$tumor cell linederived tumors were more responsive to ADC than were their TEM8- 
A
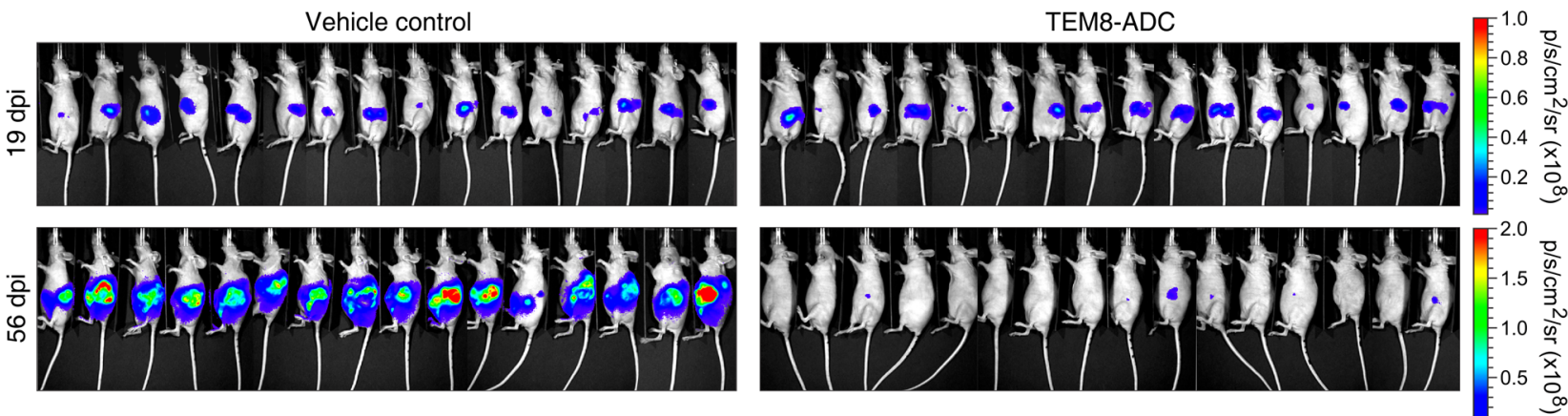

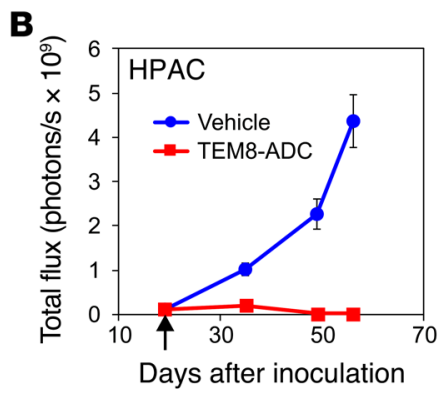

E

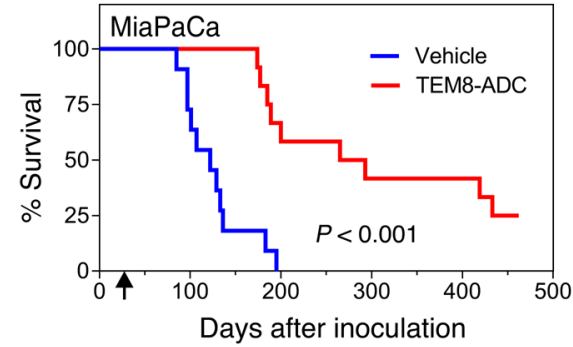

H

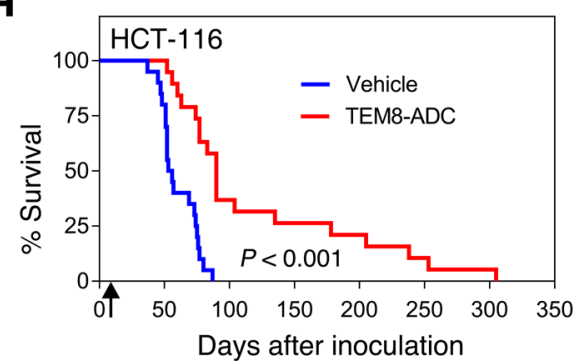

C

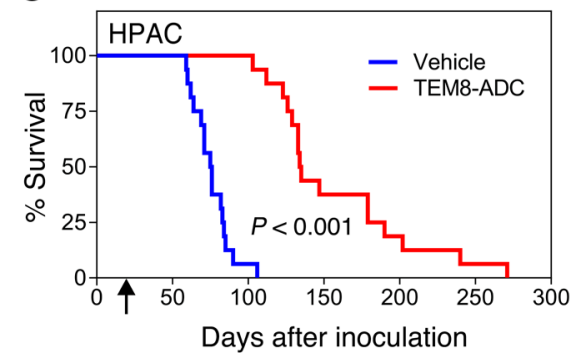

$\mathbf{F}$

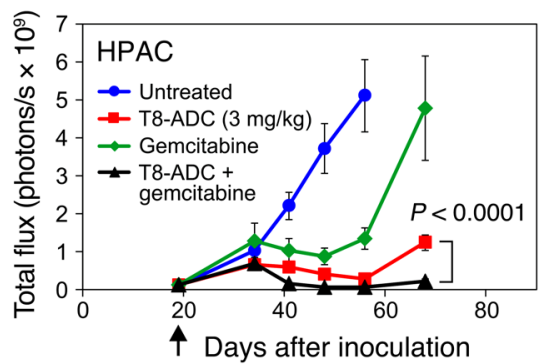

I

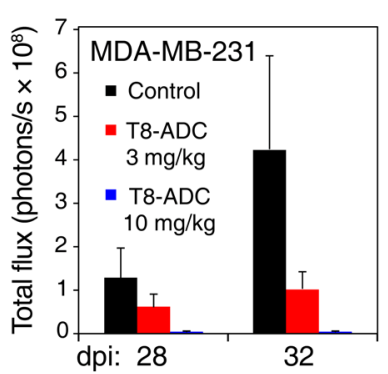

D

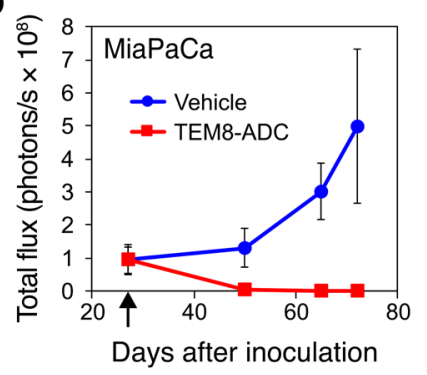

G

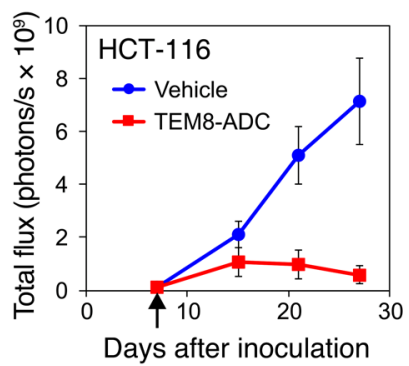

Figure 4. m825-MMAE blocks orthotopic pancreatic tumor growth as well as established colon and breast cancer metastases. (A) BLI of tumors following orthotopic injection of HPAC-luc pancreatic cancer cells into the pancreas. At 19 dpi, the mice were sorted into 2 groups of equal average tumor burden, and treatments with PBS (vehicle) or $10 \mathrm{mg} / \mathrm{kg}$ m825-MMAE (TEM8-ADC) were given twice weekly for 3 weeks. (B) Quantification of tumor burden from the HPAC study shown in A. Data represent the mean \pm SEM. (C) Kaplan-Meier survival analysis of the HPAC study shown in A. $P<0.001$, for m825-MMAE versus vehicle, by log-rank analysis. $n=16$ /group. (D) BLI was used to monitor orthotopic MiaPaCa-luc pancreatic tumor burden. In this study, mice were randomized and treatments initiated $27 \mathrm{dpi}(10 \mathrm{mg} / \mathrm{kg}$ TEM8-ADC; twice weekly for 3 weeks). $P=0.003$, by Student's $t$ test, for m825-MMAE versus vehicle 65 dpi. $n=12$ /group. (E) Kaplan-Meier survival analysis of the MiaPaCa study shown in $\mathbf{D}$. $P<0.001$ m825-MMAE versus vehicle, by log-rank analysis. $n=11$ /group. (F) BLI was used to monitor orthotopic HPAC-luc pancreatic tumor burden following treatment with $3 \mathrm{mg} / \mathrm{kg}$ TEM8-ADC (twice weekly for 3 weeks), $30 \mathrm{mg} / \mathrm{kg}$ gemcitabine (thrice weekly for 2 weeks), or a combination of both agents. (G) BLI was used to monitor HCT-116-luc colon tumor liver metastases in mice following intrasplenic injection of tumor cells. BLI was used 7 dpi to randomize mice into vehicle or $10 \mathrm{mg} / \mathrm{kg}$ TEM8-ADC treatment groups. (H) Kaplan-Meier survival analysis of the HCT-116 study shown in G. $P<0.001$, for m825-MMAE versus vehicle, by log-rank analysis. $n=19$ or $20 /$ group. Arrows in B-H indicate the day of treatment initiation. (I) BLI was used to monitor MDA-MB-231-luc breast tumor lung metastases in mice following i.v. injection of tumor cells. Mice were randomized into vehicle or $3 \mathrm{mg} / \mathrm{kg}$ or $10 \mathrm{mg} / \mathrm{kg}$ TEM8-ADC treatment groups $7 \mathrm{dpi}$. Data represent the mean $\pm \mathrm{SEM}$. $P=0.03$, by Student's $t$ test, for $10 \mathrm{mg} / \mathrm{kg}$ versus 3 mg/kg m825-MMAE 32 dpi. $n=14$ /group. 

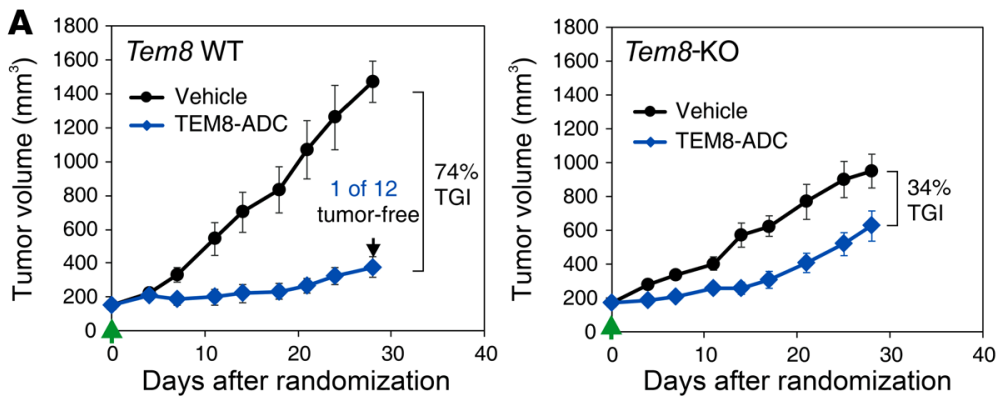

B
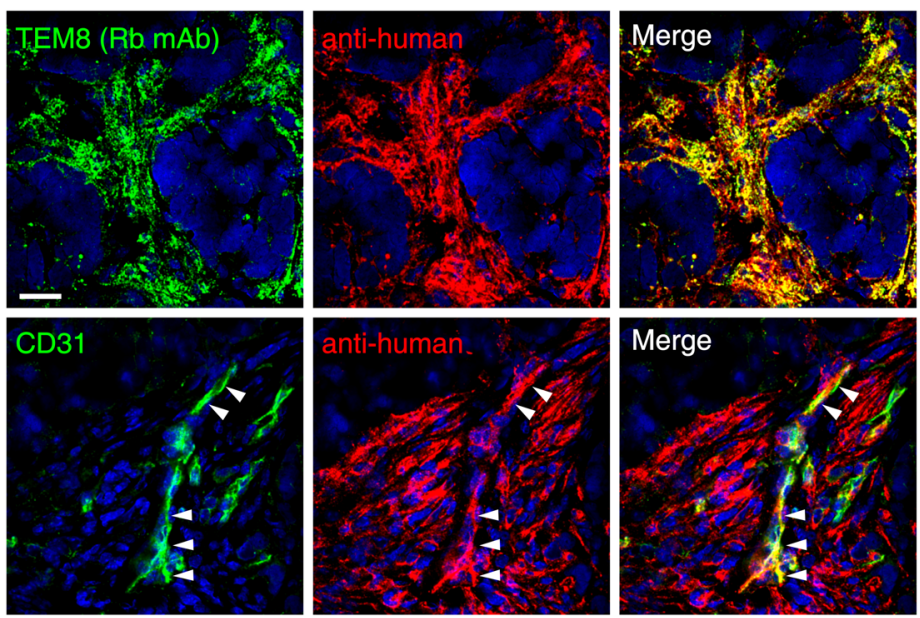

C
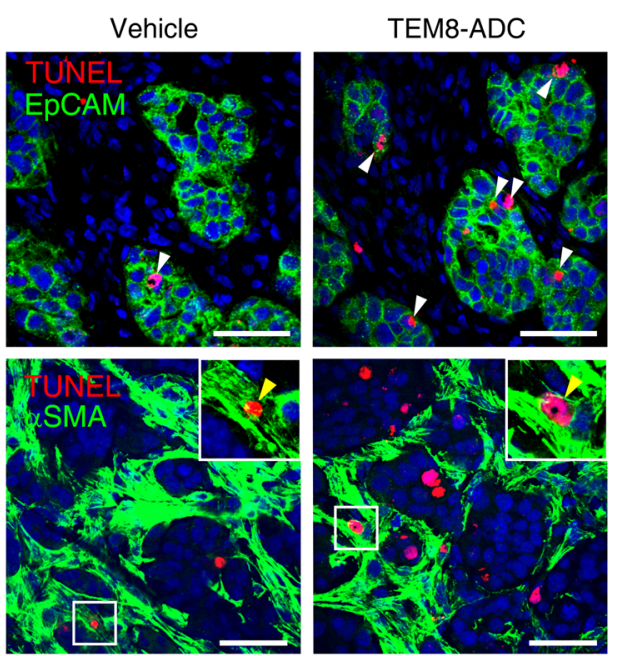

D
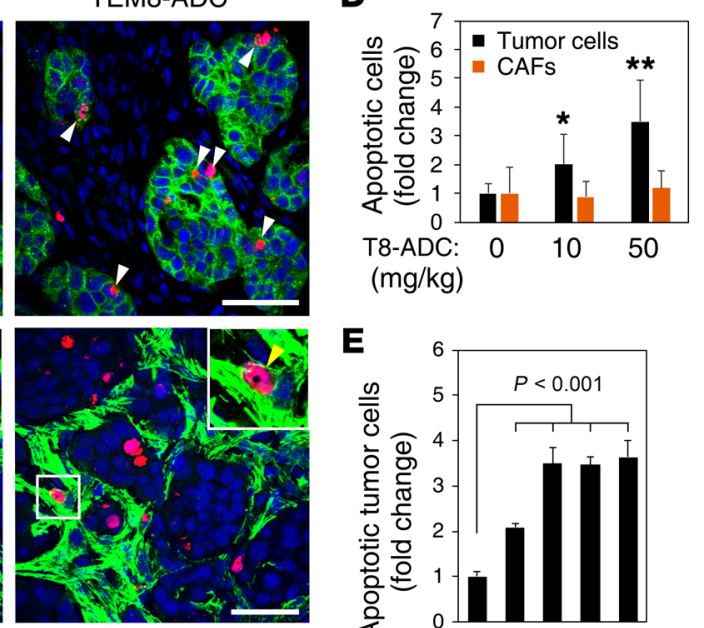

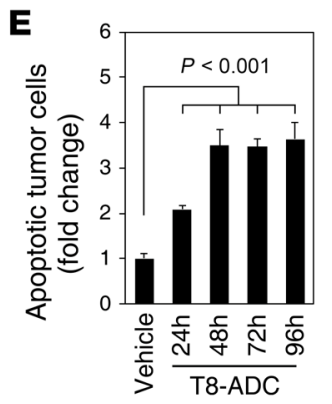

Figure 5. TEM8 expression in stromal cells is required for TEM8-ADC tumor cell killing in vivo. (A) Growth of s.c. human colon HT29 tumor xenografts in Tem8 WT and Tem8-KO mice. Treatments with vehicle or $10 \mathrm{mg} / \mathrm{kg}$ m825-MMAE (TEM8-ADC) were initiated when tumors reached an average size of $150 \mathrm{~mm}^{3}$ (green arrows). (B) Co-IF staining was used to monitor the localization of m825-MMAE (red) in HT29 tumors 24 hours after i.v. injection. The i.v. injected m825-MMAE was detected in post-staining tissue sections with Texas red-labeled anti-human secondary antibodies. Total TEM8 in tumors was detected by staining with rabbit anti-TEM8 mAb (green). Bottom panel shows m825 colocalization with CD31+ endothelium (green). Arrowheads highlight regions of colocalization (yellow in the merged image). Scale bar: $20 \mu \mathrm{m}$. (C) IF staining was used to detect TUNEL ${ }^{+}$apoptotic cells (red) 48 hours after treatment of orthotopic HPAC-T8 $8^{-1-}$ tumors with vehicle or $50 \mathrm{mg} / \mathrm{kg}$ TEM8-ADC. Tumor epithelial cells were labeled with human-specific anti-EpCAM antibodies (green; top), and CAFs were labeled with $\alpha$-SMA antibodies (green; bottom). White and yellow arrowheads (insets) indicate double-positive tumor cells and CAFs, respectively. Scale bars: $50 \mu \mathrm{m}$; original magnification, $\times 40$ (insets). (D) Quantification of TUNEL+EPCAM+ HPAC tumor cells and TUNEL ${ }^{+} \alpha-S M A^{+}$ CAFs 48 hours after treatment with 0,10 , and $50 \mathrm{mg} / \mathrm{kg}$ TEM8-ADC. Data represent the mean \pm SD. ${ }^{*} P=0.0005$ and ${ }^{*} P<0.0001$, by Student's $t$ test. (E) Quantification of TUNEL+EpCAM+ $\mathrm{HT}^{+} 9$ tumor cells 24 to 94 hours after treatment with $50 \mathrm{mg} / \mathrm{kg}$ TEM8-ADC. Data represent the mean \pm SD. The $P$ value in $\mathbf{E}$ was determined by Student's $t$ test. counterparts, with $79 \%$ of HPAC-challenged mice being tumor free following treatment compared with $60 \%$ of those challenged with HPAC-T8 ${ }^{--}$tumors (Figure 3F). Likewise, 55\% of DMS-273-challenged mice exhibited tumor eradication following treatment compared with only $22 \%$ of DMS-273-T8 ${ }^{-/}$-challenged mice (Supplemental Figure 11). Thus, TEM8 expression in stroma is required for the majority of antitumor activity, but TEM8 expression in tumor cells can also contribute to the overall efficacy of the TEM8-ADC.

Next, we explored ADC activity against orthotopic pancreatic tumors. Luciferase-labeled HPAC cells were injected into the pancreas of athymic nude mice which, after 19 days, were random- ized into treatment arms of equal average tumor burden on the basis of bioluminescence imaging (BLI). By sacrificing mice with the strongest or weakest BLI signals and excising their tumors for measurement, the pancreatic tumor volume of the live cohort was estimated to range from approximately 200 to $600 \mathrm{~mm}^{3}$ at the time of randomization. Mice were treated with vehicle (control) or $10 \mathrm{mg} / \mathrm{kg}$ m825-MMAE (biweekly $\times 3$ ). Remarkably, despite relatively large tumor sizes at the outset, m825-MMAE significantly reduced the tumor burden $(P<0.0001)$ (Figure 4 , A and B). Survival was significantly prolonged $(P<0.0001)$, with $94 \%$ (15 of 16$)$ of mice still alive in the treatment arm after the vehicle-treated 
mice had died (Figure 4C). We obtained similar results in a second orthotopic pancreatic model (MiaPaca2; Figure 4, D and E).

m825-MMAE augments the efficacy of conventional anticancer agents and blocks metastases. We next sought to determine whether m825-MMAE could augment the activity of other anticancer agents. First, we treated established orthotopic MDA-MB-231 breast tumor xenografts with m825-MMAE and/or doxorubicin (Figure 3B). As monotherapy, $3 \mathrm{mg} / \mathrm{kg}$ m825-MMAE blocked tumor growth better than did $3 \mathrm{mg} / \mathrm{kg}$ doxorubicin. Strikingly, combination therapy significantly reduced the tumor burden compared with either monotherapy ( $P=0.01$; TEM8-ADC vs. TEM8-ADC plus doxorubicin). Next, in orthotopic HPAC pancreatic tumor xenografts, $3 \mathrm{mg} / \mathrm{kg}$ m825-MMAE combined with $30 \mathrm{mg} / \mathrm{kg}$ gemcitabine significantly reduced tumor burden compared with either monotherapy $(P<$ 0.0001; TEM8-ADC vs. TEM8-ADC plus gemcitabine) (Figure 4F). Thus, m825-MMAE can augment the activity of conventional chemotherapeutic agents, which may eventually allow improved efficacy at reduced drug doses and thereby minimize toxicities.

Widespread expression of TEM8 in anatomically diverse primary tumors and metastases led us to hypothesize that the TEM8ADC may also elicit activity against preestablished metastases. To test this, we used 2 models of established experimental metastasis. In the first, luciferase-tagged HCT-116 human colon tumor cells were inoculated intrasplenically to produce colon cancer liver metastases. Seven days post inoculation (dpi), mice were randomized into two groups of equal average tumor burden, and vehicle or $10 \mathrm{mg} / \mathrm{kg}$ m825-MMAE was administered (twice weekly for 3 weeks). We observed that $\mathrm{m} 825$-MMAE significantly reduced the tumor burden $(P<0.001)$ and significantly prolonged survival $(P<$ $0.0001)$ compared with vehicle treatment (Figure $4, G$ and $H)$. In the second model, luciferase-tagged MDA-MB-231 breast tumor cells were injected i.v. into mice to induce lung metastases. Treatment with TEM8-ADC, initiated 7 days later (twice weekly for 3 weeks), led to a significant dose-dependent reduction in tumor burden by BLI at 28 and 32 dpi (Figure $4 \mathrm{I}$ ).

m825-MMAE is well tolerated in mice. To assess TEM8-ADC effects on animal health, we performed toxicology studies in mouse models. We examined mice treated with vehicle versus 10 $\mathrm{mg} / \mathrm{kg}$ treatment (twice weekly for 3 weeks), as well as mice treated with escalating single doses of $0 \mathrm{mg} / \mathrm{kg}$ (vehicle) or $10 \mathrm{mg} / \mathrm{kg}$ or $50 \mathrm{mg} / \mathrm{kg}$ ADC. We found that serum chemistries and blood cell counts were similar in all groups, and no dose-dependent alterations were observed (Supplemental Table 1). The treated mice consumed food and socialized similarly to control animals, and their body weights were unchanged by treatment (Figure 3A). Comprehensive histopathologic analysis of 42 organs or tissues failed to reveal any abnormalities (Supplemental Figure 12), with one exception: we observed increased apoptosis in enterocytes of gastrointestinal (GI) tissues after $50 \mathrm{mg} / \mathrm{kg}$ ADC administration. TUNEL staining of GI tissues confirmed ADC-induced apoptosis in Tems WT and -KO mice, revealing that toxicity was target independent and reversible upon discontinuation of treatment (Supplemental Figure 13). We conclude that the TEM8-ADC is well tolerated at a dose of $10 \mathrm{mg} / \mathrm{kg}$.

m825-MMAE tumor cell killing depends on TEM $8^{+}$tumor stroma. To explore mechanisms of ADC killing in vivo and assess the contribution of host cells, we tested m825-MMAE against HT29 colon tumor xenografts in athymic Tem $8 \mathrm{WT}$ and -KO mice. While many tumor cell lines display low levels of TEM8 by flow cytometry, we selected HT29 because it is TEM8-, preventing any direct tumor cell targeting by TEM8 ADC. TEM8-ADC treatments were initiated once tumors reached an average size of $100 \mathrm{~mm}^{3}$. Although HT29 tumors grew slower in TEM8-KO mice than in TEM8 WT mice, as expected, given the results of previous studies $(23,29)$, following ADC treatments, we observed $74 \%$ tumor growth inhibition (TGI) in Tems WT mice but only 34\% TGI in Tem8-KO mice (Figure 5A). The decrease in tumor growth in Tem8-KO mice was presumably caused by MMAE free drug, which is slowly cleaved from the ADC by carboxylesterase 1C present in mouse serum (38). Nevertheless, the increased activity of m825-MMAE observed in Tem 8 WT versus -KO mice supports the idea that stroma plays a role in mediating TEM8-ADC activity in vivo.

m825-MMAE targets TEM8 $8^{+}$stroma and induces bystander killing of tumor cells. To visualize intratumoral m825-MMAE localization, HT29 tumors were harvested 24 hours after m825-MMAE treatment and stained with anti-human antibodies to detect the fully human ADC. Costaining with rabbit anti-TEM8 mAb revealed colocalization with $\mathrm{m} 825 \mathrm{MMAE}$, indicating that the $\mathrm{ADC}$ reached most $\mathrm{TEM}^{+}$stromal cells within the tumor (Figure 5B). TEM8-ADC bound $\mathrm{CD} 31^{+}$tumor endothelium and $\mathrm{FAP}^{+} \mathrm{CAFs}$ in mice (Figure 5B and Supplemental Figure 14), revealing a staining pattern similar to that observed in ex vivo-stained human colorectal tumors (Figure 1, C and D). However, TEM8-ADC was not found in normal tissues (Supplemental Figure 15), consistent with previous studies using naked TEM8 antibodies (23).

To determine which cells were responsive to TEM8-ADC, we performed TUNEL staining on tumors 24-96 hours after administration of a single dose of 10 or $50 \mathrm{mg} / \mathrm{kg}$ m825-MMAE. Two TEM8 $8^{-}$tumor cell lines, HPAC-T8 ${ }^{-/-}$(pancreatic) and HT29 (colon), were implanted orthotopically or s.c., respectively. Surprisingly, we found that $\alpha-\mathrm{SMA}^{+} \mathrm{CAFs}$ were highly resistant to TEM8-ADC, and in both models we detected a notable fraction of $\alpha$-SMA ${ }^{-}$apoptotic cells by 24 hours after ADC treatment (Figure 5, $\mathrm{C}-\mathrm{E})$. We identified the apoptotic cell population as tumor cells by using human-specific epithelial cell adhesion molecule (EpCAM) antibodies. Tumor cell apoptosis increased by 24 hours and plateaued 3.5-fold above vehicle control levels (Figure 5E). Because the tumor cells were TEM8-, tumor cell killing suggested that m825-MMAE worked through bystander killing in vivo. Microvessel and CAF density were not altered by TEM8-ADC at these time points (Supplemental Figure 16), suggesting that the rapid onset of tumor cell apoptosis probably drove the tumoricidal responses.

Drug activation and release through stroma drives bystander killing. Given the unexpectedly rapid target-independent tumor cell killing observed in vivo, we designed a serial in vitro conditioned media $(\mathrm{CM})$ transfer assay to better understand the mechanism of tumor cell killing (Figure 6A). The assay involved 2 cell types: TEM8- HT29 tumor cells and TEM8 ${ }^{+}$tumor stromal cells (TSCs) isolated from HT29 tumors using anti-TEM8 magnetic beads. We treated the TEM8 ${ }^{+} \mathrm{TSC}$ with $\mathrm{ADC}$, followed by transfer of the CM to TEM8- HT29 cells, which were then monitored for changes in cell viability (Figure 6, A and B). Importantly, direct m825-MMAE treatment did not impact the viability of either cell type (Figure 6C). However, CM from TSCs that had been treated with ADC for 
A

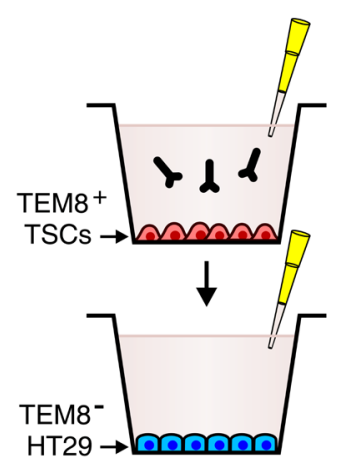

B
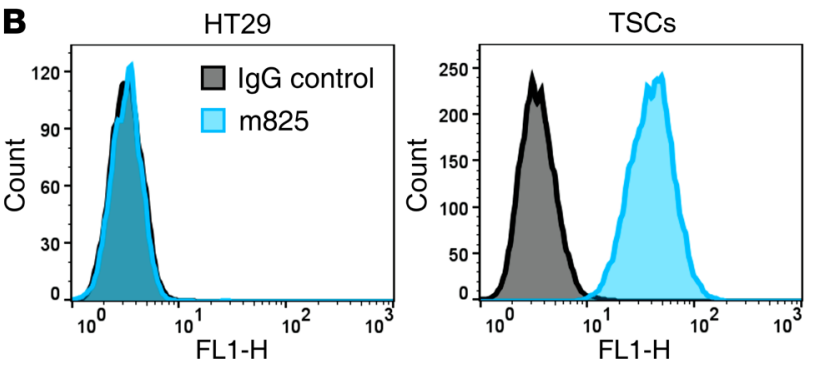

C

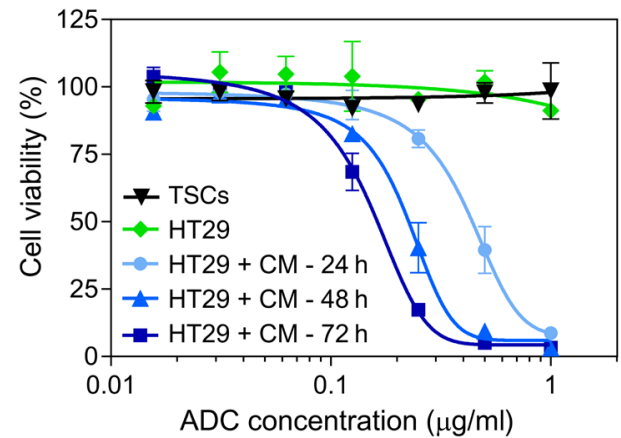

D

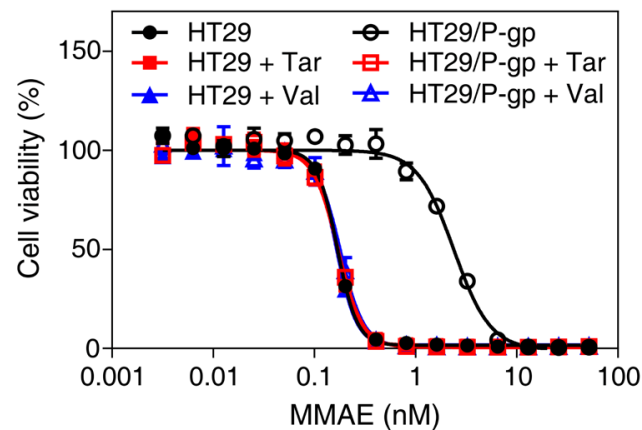

E

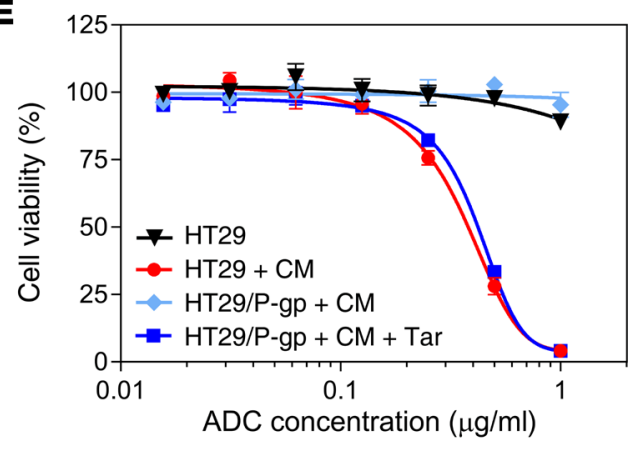

$\mathbf{F}$

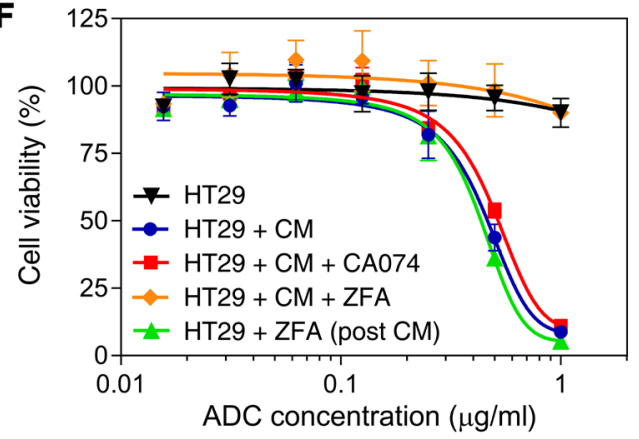

G

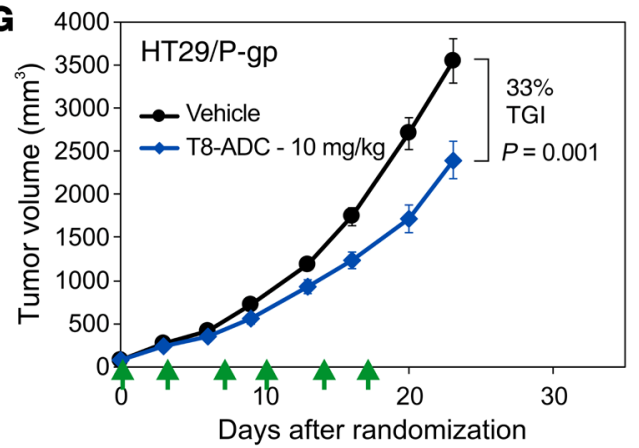

H

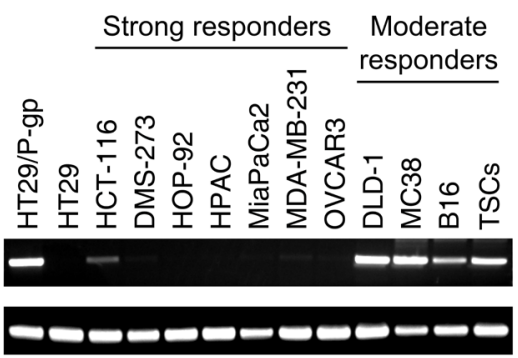

Figure 6. m825-MMAE activation by TSCs drives bystander killing. (A) In vitro CM transfer assay. (B) Flow cytometric analysis of TEM8 expression on HT29 tumor cells and TSCs using m825. (C) HT29 viability following treatment with CM from ADC-treated TSCs. CM were generated by exposing TSCs to m825-MMAE for 24 hours, 48 hours, or 72 hours. Additional controls included HT29 tumor cells or TSCs treated with nonconditioned m825-MMAE. Data represent the mean \pm SEM. (D) Impact of MMAE on the viability of HT29 and HT29/P-gp tumor cells in the presence of P-gp inhibitors $50 \mathrm{nM}$ tariquidar (Tar) or $900 \mathrm{nM}$ valspodar (Val). Data represent the mean \pm SEM. (E) Impact of TSC T8-ADC CM and $50 \mathrm{nM}$ tariquidar on HT29 and HT29/Pg-p tumor cell viability. Nonconditioned m825-MMAE was tested against HT29 tumor cells as a negative control. Data represent the mean \pm SEM. (F) HT29 cell viability following treatment with TSC TEM8-ADC CM prepared in the presence of $20 \mu \mathrm{M}$ CA074, an extracellular cathepsin B inhibitor, or $20 \mu \mathrm{M}$ Z-FA-FMK (ZFA), an intracellular cathepsin B inhibitor. As a control, $20 \mu \mathrm{M}$ ZFA was also added to HT29 cells with TSC-CM after m825-MMAE activation. Data represent the mean \pm SEM. (C) Growth of s.c. HT29/P-gp tumors. Treatments with vehicle or m825-MMAE (T8-ADC) (green arrows) were initiated when tumors reached approximately $100 \mathrm{~mm}^{3} . n=15$ /group. Data represent the mean \pm SEM. (H) RT-PCR was used to evaluate $A B C B 1 \mathrm{mRNA}$ expression in all reported cell lines. EIF4H was used as a loading control. 
Table 2. Tumor cell stratification based on MMAE responses

\section{IC50 (pM)}

\begin{tabular}{lcccc}
$\begin{array}{l}\text { Cell lines } \\
\begin{array}{l}\text { Strong responders } \\
\text { HCT-116 }\end{array}\end{array}$ & Control & Tariquidar & PSC833 & P-gp \\
\hline MDA-MB-231 & 360 & 347 & 325 & $-/+$ \\
\hline DMS-273 & 426 & 413 & 353 & - \\
\hline HT29 & 328 & 324 & 341 & - \\
\hline HOP92 & 168 & 168 & 165 & - \\
\hline OVCAR3 & 165 & 151 & 146 & - \\
\hline HPAC & 61 & 63 & 67 & - \\
\hline MiaPaCa2 & 100 & 52 & 113 & - \\
\hline Moderate responders & 101 & 128 & 105 & - \\
\hline DLD-1 & & & & \\
\hline MC38 & 2,422 & 512 & 562 & +++ \\
\hline B16 & 6,043 & 440 & 565 & +++ \\
\hline Other resistant cells & 1,469 & 558 & 516 & ++ \\
\hline HT29/MDR1 & & & & \\
\hline TSC & 2,400 & 171 & 179 & +++ \\
\hline
\end{tabular}

up to 72 hours caused a striking decrease in HT29 viability that correlated with conditioning time, i.e., the 72-hour CM were the most toxic, followed by 48-hour and 24-hour CM (Figure 6C). m825-MMAE was rapidly internalized into endosomes upon binding TSCs (Supplemental Figure 17), and ADC-TSC binding was required for cell killing, as cytotoxicity was blocked by the treatment of TSCs with an excess of unlabeled $\mathrm{m} 825$. We reasoned that m825-MMAE may have stimulated the TSCs to secrete a factor toxic to HT29 cells, or that the m825-MMAE conjugate was being cleaved by the TSCs, leading to the release of membrane-permeable MMAE free drug. Heating the ADC CM to $95^{\circ} \mathrm{C}$ for $10 \mathrm{~min}-$ utes did not reduce its cytotoxic activity, suggesting that the transferred cytotoxic factor was thermally stable. Mass spectrometry revealed free MMAE in TSC-ADC CM, indicating that cytotoxicity most likely resulted from ADC processing by TSCs followed by MMAE-mediated bystander killing of HT29 cells. Free MMAE had a fragment ion spectrum identical to that of synthetic MMAE, indicating that the ADC was cleaved at the valine-citrulline dipeptide linker, releasing MMAE free drug (Supplemental Figure 18).

Recent studies from our laboratory (39) and others $(40,41)$ suggest that MMAE can be exported from cells by the transmembrane ATP-binding cassette drug transporter P-glycoprotein (P-gp, also known as ABCB1 and MDR-1). We compared the viability of parent HT29 cells with P-gp-transfected HT29/P-gp cells and found that $\mathrm{P}$-gp conferred resistance to both MMAE free drug and TSC-ADC CM (Figure 6, D and E). Furthermore, treatment of HT29/P-gp cells with tariquidar or valspodar (PSC833), potent inhibitors of P-gp, resensitized the cells to both MMAE and TSC-ADC CM. To determine whether uptake and processing of the ADC by cathepsin B was necessary to produce free MMAE, we treated TSCs with both cell-permeable (Z-FA-FMK) and cellimpermeable (CA074) cathepsin B inhibitors prior to the ADC CM assay. While m825-MMAE was cytotoxic to HT29 if pretreated with soluble recombinant cathepsin B and this activity could be blocked by both inhibitors, only cell-permeable inhibitor blocked cytotoxicity induced by the TSC-ADC CM (Figure 6F).

To directly test the importance of bystander killing in vivo, we next treated HT29 and HT29/P-gp tumors with TEM8-ADC. We found that HT29/P-gp tumors had only 33\% treatment-induced growth inhibition (Figure 6G) compared with 92\% for HT29 tumors (Figure 3G), indicating that P-gp expression in TEM8tumor cells reduces the efficacy of the stromal-targeted ADC. Taken together, these studies indicate that TEM8-ADC kills tumor cells by drug activation and release through stroma (DAaRTS), wherein ADC is captured by tumor-associated stromal cells, the drug is cleaved, and membrane-permeable MMAE is released to kill neighboring tumor cells.

ADC tumor response relates to tumor cell MMAE sensitivity and P-gp levels. Because DAaRTS ultimately results in tumor cell killing, we reasoned that inherent sensitivity of tumor cells to MMAE may be a major determinant of ADC activity in vivo. To test this idea, we grouped cell line-derived tumors into 2 categories: those that showed a strong response to the ADC in vivo (8 tumors, Figure 3, A-G, and Figure 4D), and those that displayed a moderate response (3 tumors, Figure 3, H and I, and Supplemental Figure 6). In strong responders (defined as tumors that often regressed in response to treatment and included at least some animals in the cohort with tumor eradication), treatment with m825-MMAE showed superior activity compared with an equivalent amount of m825 naked antibody (Figure 3, A-C). Moderate responders, on the other hand, were defined as tumors that generally displayed ADC-induced tumor growth delay, with no complete tumor regressions and no discernible difference in efficacy between m825-MMAE and m825 naked antibody (Figure 3, H and I). Next, we compared in vitro sensitivity of all the tumor cell lines with MMAE free drug. Strikingly, we found that the strong responders to the ADC in vivo also had high MMAE sensitivity in in vitro cell viability assays $\left(\mathrm{IC}_{50}<1 \mathrm{nM}\right)$ (Table 2). In contrast, the moderate in vivo responders were resistant to MMAE in vitro $\left(\mathrm{IC}_{50}>1 \mathrm{nM}\right)$.

We evaluated P-gp levels in these tumor cell lines to determine whether this could explain the variation in MMAE sensitivity. Strikingly, reverse transcription PCR (RT-PCR) revealed robust $A B C B 1$ mRNA expression in all moderate responders, whereas $A B C B 1$ was undetectable or barely detectable in strong responders (Figure 6H). Treatment with tariquidar or valspodar had a negligible effect on strong responders but sensitized moderate responder cell lines to MMAE (Table 2), indicating that P-gp was likely responsible for their subdued response in vivo. Importantly, HT29 tumors that eventually progressed following TEM8-ADC treatment in vivo maintained low $A B C B 1$ levels and sensitivity to further treatment with TEM8-ADC (Figure 3G and Supplemental Figure 19). However, murine TSCs and normal human fibroblasts expressed intrinsically high $A B C B 1$ mRNA levels and were resistant to MMAE (Table 2, Figure 6H, and Supplemental Figure 20), suggesting that P-gp may also contribute to the lack of CAF apoptosis in vivo (Figure 5, C and D).

Given our data, we hypothesized that tumor cell P-gp might predict tumor responsivity to TEM8-ADC. To test this and determine whether we could identify a highly responsive immunocompetent tumor model, we evaluated $A b c b 1$ levels in 15 murine tumor 
models. Although P-gp (ABCB1) is expressed at low or undetectable levels in the tumor cells of many human cancers, including the majority of lung, pancreatic, and breast cancers (42) (Supplemental Figure 20A), consistent with our earlier analysis (Figure $6 \mathrm{H}$ ), surprisingly, we found that most murine tumor models (14 of 15) expressed high $A b c b 1$ levels (Supplemental Figure 20B). One exception was RENCA, in which $A b c b 1$ levels were undetectable. We then performed tumor studies of RENCA and $2 \mathrm{P}^{-g p^{+}}$tumors (4T1 and LLC). As predicted, on the basis of our analysis, RENCA was the only highly responsive tumor, with objective responses in all 13 mice, including 3 complete responses (Supplemental Figure 20C). In contrast, we observed modest growth delays (35\%-47\%) with tumors derived from the P-gp ${ }^{+}$tumor cell lines. These studies demonstrate that it may be possible to predict tumor responsiveness to TEM8-ADC therapy on the basis of tumor cell P-gp levels.

\section{Discussion}

Although the tumor microenvironment is known to play an indispensable role in tumor growth, potential vulnerabilities afforded by stromal cells remain unexploited, in part due to a lack of recognized suitable cancer-associated stromal cell targets. Here, we demonstrate broad overexpression of TEM8 in tumor-associated stromal cells, predominantly in CAFs, endothelium, and pericytes. Given the established role of stroma in promoting tumorigenesis $(23,29)$, our initial goal was to deplete tumors of TEM ${ }^{+}$ tumor stroma through TEM8-ADC treatment. Instead, we found that ADC efficacy was driven through an unexpected mechanism, which we call DAaRTS, whereby drug-resistant TEM8 ${ }^{+}$tumor stromal cells bind, internalize, and process ADC, releasing cellpermeable MMAE free drug to induce rapid bystander killing of nearby drug-sensitive tumor cells in a target-independent manner.

Most ADCs depend on direct tumor cell binding for activity, whereas DAaRTS exploit the tumor microenvironment for ADC prodrug activation. While tumor cell-directed ADCs frequently target only a limited number of tumor types or a subset of patients with defined alterations (e.g., trastuzumab emtansine targeting HER2 ${ }^{+}$tumors), ADCs that react with tumor-associated stroma can potentially target many different tumor types. TEM8 was found to be broadly expressed throughout the stroma of most primary tumors and metastases in both humans and mice. TEM8-ADC treatment resulted in regression and often eradication of multiple tumor types, including lung and breast cancer PDX models. Furthermore, TEM8-ADC showed activity against preestablished metastasis of human colon and breast cancer, prolonging overall survival. By exploiting the resistance of $\mathrm{P}-\mathrm{gp}^{+}$host stromal cells, free drug could be localized at the tumor site, which was sufficient, in many cases, to eradicate tumors and prevent acquired resistance.

Recent studies have highlighted a correlation between tumor cell P-gp levels and resistance to MMAE-linked ADCs (41). Here, by genetically engineering MMAE-sensitive tumor cells to express P-gp, we directly demonstrate that this multidrug pump contributes to tumor cell resistance both in vitro and in vivo, in agreement with findings from another recent study (40). Two P-gp- tumor models, MDA-MB-231 and OVCAR-3, were sensitive to the TEM8-ADC, even though these tumors were derived from patients who had relapsed following combination chemotherapy with adriamycin $(43,44)$. These results highlight the notion that in vivo resistance to $\mathrm{P}$-gp-sensitive drugs can occur without P-gp induction and that certain chemoresistant tumors may retain sensitivity to TEM8-ADC. However, because other tumors may acquire or intrinsically express P-gp, assessing P-gp expression in tumor cells could represent a useful diagnostic biomarker to guide patient selection and ensure that the TEM8-ADC is given to those most likely to respond. Moreover, because MMAE, DM-1, and calicheamicin are all substrates of P-gp $(45,46)$, these results have potential implications for clinically approved ADCs and many others in clinical development. Identifying alternative payloads that are insensitive to drug-efflux pumps like P-gp could be particularly valuable for patients whose tumor cells are P-gp .

A major concern for any ADC approach is the potential for adverse effects. Many ADCs currently in clinical development have been designed to target human tumor cell antigens and do not cross-react with corresponding mouse orthologs, limiting preclinical toxicology studies. We designed fully human antibodies that are cross-reactive with both mouse and human TEM8, allowing us to assess on-target/off-tumor toxicities in our preclinical mouse models. We found that m825-MMAE was well tolerated, with no overt alterations in body weights or other clinical parameters. Extra attention was given to normal brain, lung, and kidney, in which we detected low levels of TEM8 in some cases, but observed no toxicities in these tissues. One possible explanation is that the expression noted in normal adjacent clinical samples was induced by nearby tumor tissue. Also, MMAE is known to selectively target actively dividing cells, providing a safeguard that helps protect quiescent "normal" cells. Because ADC activity is also dependent on receptor levels (39), another possibility is that TEM8 levels in normal cells lie below the threshold needed for cytotoxic activity. Histopathological analysis revealed mild off-target toxicities in the GI tract following administration of 50 $\mathrm{mg} / \mathrm{kg}$ TEM8-ADC, a dose 5 times that used in our efficacy studies. However, because these toxicities were target independent, they were probably caused by the premature release of MMAE from the ADC by carboxylesterase $1 \mathrm{C}$, an enzyme present in mouse but not human serum. Moreover, the reversibility of GI toxicities upon treatment cessation suggests that any potential GI toxicities may be manageable. Further toxicity studies in primates (which lack serum carboxylesterase 1C) will be required to fully establish the safety profile of TEM8-ADC. The ADC field is also rapidly maturing, and it is possible that additional modifications involving, for example, site-specific drug conjugation and increased drug-to-antibody ratios through surface hydrophobicity masking could also help improve the therapeutic index of TEM8-ADC (47-49).

Recently, treatment with MMAE-linked ADCs has been proposed as a strategy to overcome immune suppression and augment the activity of immune checkpoint inhibitors (50,51). Direct intratumoral injection of microtubule-destabilizing agents has also been proposed as a strategy to simultaneously kill tumor cells and promote the maturation of tumor-infiltrating DCs and cytotoxic T lymphocyte (CTL) activity (52). Although the mechanisms responsible for this $\mathrm{T}$ cell potentiation remain incompletely understood $(53,54)$, DAaRTS could potentially augment immunotherapies by localizing MMAE to surgically inaccessible tumors following systemic delivery. 
In summary, we show that intrinsically resistant, tumor-associated stromal cells can be exploited to function as a drug-activating sponge, removing ADC prodrug from the circulation and focusing active free drug on the tumor. TEM8 overexpression is associated with most solid tumor types, such as breast, colon, lung, and pancreatic tumors, for which there is an urgent need of improved therapies. Widespread overexpression of TEM8 in cancers from disparate anatomical sites suggests that TEM8-ADCs may be particularly useful for the treatment of late-stage metastatic disease.

\section{Methods}

Cell lines and PDX models. The cell lines 293, CHO-K1, HPAC, MiaPaCa2, MDA-MB-231, DLD-1 CT26, LLC, MLE12, 4T1, EMT6, JC, PY230, and PY8119 were obtained from ATCC. E0771 was obtained from CH3 BioSystems. DMS-273, HOP92, HCT116, HT29, OVCAR3, B16, PANO2, PAN03, and glioma 261 cell lines and COS-G and CLO-G PDX models were obtained from the Division of Cancer Treatment and Diagnosis (DCTD) Tumor Repository of the NCI. COS-G (papillary lung carcinoma) and CLO-G (infiltrating duct breast carcinoma) were engrafted from patients into $\mathrm{nu} / \mathrm{nu}$ mice. MC38, RENCA, and CHO-PR230 (CHO) cell lines were gifts of Jeffrey Schlom (NCI, NIH), Jonathan M. Weiss (NCI, NIH), and Stephen H. Leppla (National Institute of Allergy and Infectious Diseases [NIAID]), respectively. HCT-116-luc cells were previously described (55). Culture-adapted PyMT tumor cells were derived from spontaneous tumors in the MMTV-PyMT mammary tumor model (FVB/ N-Tg[MMTV-PyVT]634Mul/J; The Jackson Laboratory). Cultureadapted PDA4 pancreatic tumor cells were obtained from the Center for Advanced Preclinical Research (CAPR) at the NCI and derived from spontaneous tumors that developed in a Kras, p53, PdxCre (KPC) pancreatic tumor model (56). Cells expressing mTEM8, hTEM8, mCMG-2, hCMG-2, FLAG-mANTXRL, FLAG-hANTXRL, hP-gp, or luciferase were generated by Lipofectamine 2000-mediated (Thermo Fisher Scientific) stable transfection.

IHC. FFPE sections were deparaffinized, treated with proteinase K, Dual Endogenous Enzyme-Blocking Reagent, biotin block (Dako), and then blocked with $1 \%$ blocking reagent (Roche) in TBS (100 mM Tris [pH 7.5], $150 \mathrm{mM} \mathrm{NaCl}$ ) plus $1 \%$ Triton-X 100. Sections were incubated with rabbit anti-human TEM8 (c37; Epitomics) for 2 hours at room temperature, followed by signal amplification (Vectastain ABC HRP Kit; Vector Laboratories).

IF staining. For co-IF staining of human colorectal tumors, TEM8 was labeled with either rabbit anti-TEM8 (c37; Epitomics) or chimeric anti-TEM8 (c-m825) primary antibody. CAFs were stained with mouse anti- $\alpha$-SMA (clone 1A4; MilliporeSigma); rat anti-FAP $\alpha$ (MABS1001; Vitatex); or rabbit anti-PDGFR $\beta$ (clone 28E1; Cell Signaling Technology) antibodies. Endothelium was stained with mouse anti-CD146 (clone P1H12; Thermo Fisher Scientific) and pericytes with goat anti-desmin (R\&D Systems). Isotype-matched nonspecific IgGs were used as controls. For in vivo ADC-imaging, $10 \mathrm{mg} / \mathrm{kg}$ m825-MMAE was injected i.v. into HT29 tumor-bearing mice, and tissues were collected 24 hours later. ADC was detected with biotin goat anti-human IgG or biotin donkey anti-human IgG secondary antibodies (Jackson ImmunoResearch Laboratories) followed by Texas Red streptavidin (Vector Laboratories) or FITC goat anti-human IgG (Jackson ImmunoResearch Laboratories) followed by 488-goat anti-FITC (Invitrogen, Thermo Fisher Scientific). For costaining, rat
anti-CD31 (clone MEC13.3; Santa Cruz Biotechnology) or rabbit antiFAP (H-56; Santa Cruz Biotechnology) primary antibodies were used. For analysis of apoptosis, vehicle or 10 or $50 \mathrm{mg} / \mathrm{kg} \mathrm{m825-MMAE}$ was injected i.v. into mice with orthotopic pancreatic (HPAC-T8-/-) or s.c. colon (HT29) tumors. Tumors were excised 24-96 hours later and stained with FITC-conjugated mouse anti-EpCAM (clone BerEP4; Dako) or FITC-conjugated mouse anti- $\alpha$-SMA antibody (clone 1A4; MilliporeSigma). Apoptotic cells were labeled with the ApopTag Red Kit (catalog S7165; MilliporeSigma). For tumor cell (or CAF) apoptosis, the number of TUNEL ${ }^{+}$cells within the $\mathrm{EpCAM}^{+}$(or $\alpha-\mathrm{SMA}^{+}$) regions was normalized to the $\mathrm{EpCAM}^{+}$(or $\alpha-\mathrm{SMA}^{+}$) surface area and presented as the fold change relative to the untreated control. CAF and vessel densities were calculated by determining the average $\alpha-\mathrm{SMA}^{+}$or $\mathrm{CD} 31^{+}$area as a percentage of the total tumor area and presented as the fold change relative to the vehicle control. Images were captured with either a Zeiss LCI510 or a LSM780 confocal microscope and analyzed using Fiji software (ImageJ, NIH).

Expression vectors. Full-length human and mouse FLAG-tagged ANTXRL expression vectors, containing an N-terminal 3×FLAG tag and codon optimized ORFs, were synthesized (IDT) and assembled by Gibson assembly (New England BioLabs) into pcDNA3.1 (Invitrogen, Thermo Fisher Scientific). The sequences for hANTXRL and mAntxrl have been deposited in GenBank (accession numbers KY947541 and KY947542).

Development of rabbit $m A b$ against TEM8. In collaboration with Epitomics, rabbits were injected sequentially with both recombinant hTEM8(ECD)-Fc protein and 293 cells stably overexpressing fulllength mouse TEM8 on the cell surface (293/mTEM8). Rabbits with the highest antibody titers were identified by comparing before and after immunization bleeds for selective reactivity with $\mathrm{CHO}$ cells stably overexpressing either mTEM8 or hTEM8. Hybridoma supernatants that displayed specific reactivity with TEM8-ECD by ELISA were screened for reactivity with native TEM8 by flow cytometry using CHO-mTEM8 and CHO-hTEM8 cells. Clones that tested positive by flow cytometry were then tested for reactivity with TEM8 in IP, immunoblotting, and IF applications. One mAb, c37 (clone 37), that reacted with both mTEM8 and hTEM8 in each of the assays was then used to generate stable 293-derived producer cells for antibody production and purification.

m825 antibody production and purification. A yeast display library was constructed using a collection of human antibody gene repertoires, including the genes used for the construction of a phage display Fab library (57) and those from more than 50 additional individuals. As this library is immune naive through in vitro stochastic pairing of VH and VL repertoires, it is not subject to tolerance mechanisms found in normal immune responses and allowed the generation of antibodies against regions of the TEM8 ECD that are $100 \%$ conserved between mouse and human. In vitro selection of the yeast display library involved 3 rounds of sequential panning on biotinylated, purified recombinant TEM8(ED)-AP and TEM8-Fc fusion proteins. Biotinylated hTEM8(ED)-AP $(10 \mu \mathrm{g})$ was incubated with $5 \times 10^{10}$ cells from the initial naive antibody library in $50 \mathrm{ml}$ PBS-BSA (PBS containing 0.1\% BSA) for 2 hours, washed with PBSBSA, and captured with streptavidin-conjugated microbeads from Miltenyi Biotec using the AutoMACS System. The sorted cells were amplified, and the panning was repeated once with hTEM8(ED)-AP and once with mTEM8(ED)-AP protein to enrich for cross-reactive binders. The highest affinity $\mathrm{mAb}, \mathrm{m} 825$, was converted into full- 
size human IgG1. As an additional research tool, we also created a mouse-human chimeric m825 (c-m825) full $\operatorname{IgG}$, wherein the constant domains of mouse IgG2a (CH1, CH2, CH3, and CL) were fused to the human variable domains (VH and VL). $\mathrm{m} 825$ or $\mathrm{c}-\mathrm{m} 825$ IgGs were collected from culture supernatants grown in serumfree medium and purified by protein A chromatography. Antibody preparations for in vivo studies possessed less than $5 \%$ aggregates and had endotoxin levels below $1 \mathrm{EU} / \mathrm{mg}$.

Preparation of m825-MMAE ADC. The m825-MMAE ADC was made by conjugating the antibody to a maleimide-activated drug-linker containing MMAE using a 2-step reaction. Partial reduction of antibody interchain disulfide bonds with tris (2-carboxyethyl)-phosphine hydrochloride (TCEP $\mathrm{HCl}$ ) yielded a reduced antibody intermediate mixture containing free thiols. In the second step, the partially reduced antibody was reacted with excess maleimide-activated drug-linker to afford the crude ADC product with the desired average drug-to-antibody ratio (DAR) of approximately 4 and ultrafiltered. The final formulation (10 $\mathrm{mg} / \mathrm{ml} \mathrm{m825-MMAE}$ in $20 \mathrm{mM}$, pH 5.8 histidine buffer) was sterile filtered. Antibody preparations used for in vivo studies had a DAR of 4, possessed $5 \%$ aggregates, and had endotoxin levels of $0.275 \mathrm{EU} / \mathrm{mg}$.

Antibody affinity measurements. m825 Fab was generated from full-size IgG1 using the Pierce Fab Preparation Kit (Thermo Fisher Scientific). Surface plasmon resonance was used to measure binding affinity of the Fab to the TEM8 ECD on a BIAcore X100 instrument (GE Healthcare). Purified mAP-TEM8 and hAP-TEM8 fusion proteins were diluted in $10 \mathrm{mM}$ sodium acetate buffer ( $\mathrm{pH}$ 5.0) and immobilized on a CM5 biosensor chip using an amine coupling kit. The running buffer was HBS-EP (10 mM HEPES, pH 7.4, 150 mM NaCl, 3 mM EDTA, 0.05\% surfactant P20). The Fabs diluted with the running buffer were allowed to flow through the cells at concentrations ranging from $0.05 \mathrm{nM}$ to $500 \mathrm{nM}$. After 10 minutes of dissociation, the chip was regenerated with $10 \mathrm{mM}$ acetate buffer, $\mathrm{pH}$ 4.0. The data were fitted with a 1:1 binding model, and the dissociation rate constant was estimated with BIAevaluation software (Biacore).

TEM8 ADC cell uptake assay. CHO-hTEM8 and TSC cells grown on poly-D-lysine-coated glass-bottomed dishes (MatTek) for 24 hours were rinsed with PBS containing 0.5\% BSA (PBS-BSA) and stained with $4 \mu \mathrm{g} / \mathrm{ml} \mathrm{m825,} \mathrm{m825-MMAE,} \mathrm{or} \mathrm{isotype-matched} \mathrm{nonspecific} \mathrm{human}$ IgG diluted in culture medium containing $0.5 \%$ BSA on ice for $30 \mathrm{~min}$ utes. Cells were rinsed with PBS-BSA and stained for an additional 30 minutes on ice with an FITC-goat anti-human secondary antibody and then either transferred to a $37^{\circ} \mathrm{C}$ incubator for 60 minutes or left on ice. Cells were then counterstained with CellMask Orange plasma membrane stain and Hoechst 33258 nucleic acid stain (both from Thermo Fisher Scientific) and imaged on a Zeiss LSM510 microscope.

IP and immunoblotting. Lysates were incubated overnight with human anti-TEM8 $\mathrm{mAb}(\mathrm{m} 825)$. The precipitated proteins were eluted from protein A agarose beads (Roche), separated by SDS-PAGE, and transferred onto a PVDF membrane (MilliporeSigma). Immunoblots were probed with either rabbit anti-TEM8 primary mAb (c37; Epitomics) followed by HRP-conjugated anti-rabbit secondary antibody; mouse anti-CMG2 primary mAb (1H8) (a gift of Stephen H. Leppla, NIAID) followed by HRP-conjugated anti-mouse secondary antibody; or HRP-conjugated mouse anti-FLAG primary $\mathrm{mAb}$ and exposed using WestDura ECL (Thermo Fisher Scientific). Immunoblots were probed with HRP-conjugated mouse anti-GAPDH primary mAb (clone 6C5; Abcam) as loading controls.
Flow cytometry. Cells were trypsinized, rinsed in cold PBS-BSA, and labeled with m825-MMAE, an isotype-matched human IgG, or FITC-conjugated protective antigen (List Biologicals Laboratories) in PBS-BSA at $4^{\circ} \mathrm{C}$. Next, cells were rinsed, incubated with FITC-conjugated goat antihuman IgG or 488-goat anti-FITC secondary antibodies, and then rinsed again. Cells transfected with FLAG-tagged ANTXRL were detached with 1 mM EDTA due to trypsin sensitivity and labeled with FITC-conjugated mouse anti-FLAG mAb followed by 488 -goat anti-FITC secondary antibody. Analysis was performed on a FACSCalibur Flow Cytometer (BD).

ELISA. TEM8 ELISA plates were prepared by coating TEM8 ECD protein at $1 \mu \mathrm{g} /$ well overnight at $4^{\circ} \mathrm{C}$ onto UltraCruz high-binding 96-well ELISA plates (Santa Cruz Biotechnology). Titrations of m825 human antiTEM8 mAb, m825-MMAE, and an isotype-matched human IgG were incubated on the plate for 1 hour, followed by biotinylated goat anti-human secondary antibody and streptavidin-HRP. Signal was detected using 1-Step Ultra TMB-ELISA substrate solution (Thermo Fisher Scientific).

$R T-P C R$. RNA was isolated from cells using the RNeasy Kit (QIAGEN), and cDNA was synthesized using the Superscript III Kit (Invitrogen, Thermo Fisher Scientific). The following PCR primer pairs were used: hANTXRL, forward: 5'-GAGCAGGAAGCCTTCGGTAC-3', hANTXRL, reverse: 5'-TGTGCCACCAGTTCTCCATC-3'; mAntx$r l$, forward: 5'-TGGTGTCATCCCAAGGCG-3', mAntxrl, reverse: 5'-TCTAAGACCTGCTACGTGTGTCC-3'; $m A b c b 1$ and $h A B C B 1$, forward: 5'-ATGGATGAGATTGAGAAAGCTGTC-3', $m A b c b 1$ and $h A B$ $C B 1$, reverse: 5'-TGACAAGTTTGAAGTAAATGCC-3'; and $m E i f 4 h$ and hEIF $4 H$, forward: 5'-GGCTAGTCAGAGACAAAGACACAG-3', mEif $4 h$ and $h E I F 4 H$, reverse: $5^{\prime}$-ATGTCCACACGAAGTGACCG-3'. The $m A b$ $c b 1$ and $h A B C B 1$ and $m E i f 4 h$ and $h E I F 4 H$ primer pairs were designed against sequences that are $100 \%$ conserved between mice and humans. hABCB1, forward: 5'-CTGGTTGCTGCTTACATTCAGG-3', hABCB1, reverse: $5^{\prime}$-GGAGTCAACGGATTTGGTCG-3'; and $h G A P D H$, forward: 5'-GGAGTCAACGGATTTGGTCG-3', hGAPDH, reverse: 5'-GAGGCATTGCTGATGATCTTG-3'. The human $h A B C B 1$ and $h G A P D H$ primer pairs were designed to react with human but not mouse genes.

m825-MMAE serum stability test. Briefly, for the m825-MMAE serum stability test (Seventh Wave Laboratories), m825-MMAE was incubated with PBS or serum from mouse, rat, monkey (cynomolgus), or humans for up to 21 days, flash frozen, and then analyzed by solid phase extraction of free MMAE followed by LC-MS/MS analysis. Auristatin $\mathrm{F}$ was used as an internal control.

Cell viability. Cell viability was measured using Alamar Blue (Thermo Fisher Scientific). Cells were plated and 24 hours later treated in triplicate with m825-MMAE, m825, MMAE free drug, or MMAE plus P-gp inhibitors (50 nM tariquidar, a gift of Michael M. Gottesman (NCI, $\mathrm{NIH}$ ); $900 \mathrm{nM}$ Valspodar, MilliporeSigma). Three to five days after treatment, $10 \%$ Alamar Blue reagent was added to the plates, and fluorescence was measured on a CLARIOstar Microplate Reader (BMG Labtech) according to the manufacturer's instructions.

Toxicology studies. C57BL/6 mice were treated with $20 \mathrm{mM}$ histidine buffer (vehicle control) or 10 or $50 \mathrm{mg} / \mathrm{kg} \mathrm{m825-MMAE.} \mathrm{Samples} \mathrm{were}$ processed 24 hours later for complete blood counts (CBC), serum chemistry, and histology. Forty-two separate tissues were evaluated from each group ( 3 males and 3 females, total of 6 mice/group) by a board-certified veterinary pathologist $(\mathrm{DCH})$. To determine long-term toxicity, these experiments were repeated using athymic NCr nude mice ( 2 males and 7 females, total of 9 mice/group). Mice were treated with $10 \mathrm{mg} / \mathrm{kg} \mathrm{m825-}$ MMAE (twice weekly for 3 weeks). For TUNEL staining in GI tissues, 
10 or $50 \mathrm{mg} / \mathrm{kg}$ m825-MMAE was injected i.p. into Tems WT or -KO C57BL6-NCr mice. The animals were euthanized 24 hours to 14 days later, GI tissue was frozen, and immunofluorescence TUNEL staining was performed as described above.

Tem8 CRISPR-Cas9. TEM8 guide RNAs in pCas-Guide-GFP (Santa Cruz Biotechnology) were transfected with Polyfect (QIAGEN) into TEM8 ${ }^{+}$DMS-273 or HPAC cells. Transfected cells were sorted by FACS 48 hours later, expanded, and then sorted again. The resultant cells were stained with $\mathrm{m} 825$ followed by PE-labeled goat anti-human IgG1, and PE- single cells were sorted by FACS into individual wells of 96-well plates and the clones expanded. KO status was confirmed by FACS and DNA sequencing. As DMS-273 TEM 8 WT cells are a pooled population, $16 \mathrm{KO}$ clones were combined to generate TEM8-KO pools.

Isolation of immortalized TSCs. TSCs were isolated from HT29 tumors grown in SCID mice that contained a temperature-sensitive SV40 TAg transgene (Immortomouse, The Jackson Laboratory) (58). TEM8 ${ }^{+}$TSCs were isolated using biotinylated anti-TEM8 antibodies (m825) conjugated to streptavidin-linked magnetic beads (20). Cells were expanded for 2 weeks at $33^{\circ} \mathrm{C}$ and cloned by limiting dilution. TSCs were maintained at $33^{\circ} \mathrm{C}$ in DMEM media supplemented with IFN- $\gamma$ and only shifted to $37^{\circ} \mathrm{C}$ (to silence the immortalizing SV40 TAg transgene) for experiments.

Tumor studies. Tumor studies were performed in 7- to 10-week-old female C57BL6-NCr (B16, MC38, LLC), BALB/c (4T1, RENCA), athymic NCr-nu/nu (DLD-1, DMS-273, HCT-116, HT29, HOP92, HPAC, MDA-MB-231, MiaPaCa2, COS-G, CLO-G), or CB17 SCID (OVCAR3) mice (Charles River Laboratories). For tumor studies involving Tem8-KO mice, athymic nude Tem $8 \mathrm{WT}$ or Tem 8 -KO littermates derived from Tem 8 heterozygous intercrosses (23) were randomly assigned to experimental groups. Tumor cells $\left(2 \times 10^{6}\right.$ to $\left.5 \times 10^{6}\right)$ were injected into the mammary fat pad (4T1 and MDA-MB-231) or s.c. into the flank. HPAC-luc or MiaPaCa2-luc tumor cells $\left(4 \times 10^{5}\right)$ were coinjected with Matrigel (3:2 with PBS) into the pancreas. To produce liver metastases, $3 \times 10^{6}$ HCT-116-luc tumor cells were injected into the spleen. After 5 minutes (to allow liver seeding), the spleens were resected and the abdomens sutured. To produce lung metastases, $1 \times 10^{6}$ MDA-MB-231-luc tumor cells were injected into the tail vein. For the PDX models, $2 \mathrm{~mm}^{2} \mathrm{COS}-\mathrm{G}$ or $1 \mathrm{~mm}^{2} \mathrm{CLO}-\mathrm{G}$ tumor tissue fragments were implanted s.c. or into the mammary fat pad, respectively. Subcutaneous and mammary fat pad tumors were measured with a digital caliper, and tumor volumes were calculated using the formula $\mathrm{L} \times \mathrm{W}^{2} \times 0.5$ and presented as the mean \pm SEM. Orthotopic pancreatic tumors and liver and lung metastases were measured using BLI. For therapeutic studies, mice were sorted into groups containing an equal average tumor size (usually $100 \mathrm{~mm}^{3}$ ) immediately prior to drug administration. To minimize variation, mice were excluded from the analysis at the time of sorting if their tumors were less than half the average tumor size for the group or more than double the average tumor size. Tumor measurements were taken by technicians blinded to the objectives of the study. The survival endpoint was reached when mice became moribund or lost more than $20 \%$ of their body weight. Mice were treated with vehicle (20 $\mathrm{mM}$ histidine), TEM8 antibodies, or free drug at the doses and schedules indicated in Figure 3, Figure 4, B-I, Figure 5A, and Figure 6G. ADC was initially administered i.v. by tail vein injection, but during this work we found that i.v. and i.p. dosing produced equivalent results (Figure 3C).

CM transfer assay. CM were collected from confluent TSCs in 6-well plates and exposed to m825-MMAE for 24 to 72 hours. HT29 tumor cells and TSCs were plated at 1,000 cells/well into 96-well plates, incubated for 24 hours, and then treated in triplicate with
m825-MMAE or TSC CM. For MMAE free drug-specific killing, HT29/P-gp tumor cells were treated with TSC CM or TSC CM plus 50 $\mathrm{nM}$ tariquidar. To evaluate intracellular cathepsin B-dependent cleavage, CM were collected from TSCs exposed to m825-MMAE plus 20 $\mu$ M Z-FA-FMK (Santa Cruz Biotechnology) or to $20 \mu$ M CA074 (R\&D Systems) for 72 hours. HT29 tumor cells were then treated with inhibitor-exposed TSC CM or control TSC CM without the inhibitors, and Z-FA-FMK was added after conditioning. After 4 days, viability was measured using Alamar Blue as described above.

Statistics. A Student's $t$ test was used to calculate differences in tumor volumes between 2 groups. For Kaplan-Meier survival analysis, a log-rank (Mantel-Cox) test was used to compare the study arms. Differences between 2 groups are presented as the mean \pm SEM or the mean \pm SD. Sample sizes $(n)$ are indicated in the figure legends. All tests were 2 -sided, and $P$ values of less than 0.05 were considered statistically significant. Statistical analysis was performed using GraphPad Prism 6.04 (GraphPad Software).

Study approval. Anonymized human clinical samples were obtained from US Biomax or the Cooperative Human Tissue Network (CHTN) with approval from the NIH Office of Human Subject Research. Frederick National Laboratory is accredited by the Association for Assessment and Accreditation of Laboratory Animal Care (AAALAC International) and follows the Public Health Service Policy for the Care and Use of Laboratory Animals. Animal care was provided in accordance with the procedures outlined in the Guide for the Care and Use of Laboratory Animals (National Academies Press, 2011) and protocols approved by the IACUC of the NCI.

\section{Author contributions}

CS, S. Saha, XMZ, ZZ, DSD, and BSC conceived and designed the study. CS, XMZ, ZZ, S. Seaman, DW, GD, AC, EZ, DSD, and BSC were responsible for methodology development. CS, ZZ, MBH, KM, S. Seaman, JMD, KSH, GJY, HM, DCH, YW, JH, YF, AC, EZ, and BSC performed data acquisition, analysis, and/or interpretation. CS and BSC wrote the original draft of the manuscript. All authors contributed to the writing, review, and/or revision of the manuscript. XMZ, ZZ, DAS, DCH, DSD, and BSC supervised the study.

\section{Acknowledgments}

We thank Michael M. Gottesman (NCI, NIH) for advice and P-gp plasmids and inhibitors and Bernard Violand (consultant to BioMed Valley Discoveries), David Tung, Linping Zhang, and Halle Zhang (all from BioMed Valley Discoveries) for helpful discussions. This work was supported by a METAvivor grant and a Cooperative Research and Development Agreement (CRADA) between BioMed Valley Discoveries and the NCI, NIH. Federal funds were also provided by the NCI, NIH under contract no. HHSN261200800001E, and the Center for Cancer Research (CCR), part of the NCI's intramural research program, NIH, Department of Health and Human Services (DHHS). The content of this publication does not necessarily reflect the views or policies of the DHHS, nor does mention of trade names, commercial products, or organizations imply endorsement by the US government.

Address correspondence to: Brad St. Croix, P.O. Box B, Building 560, 1050 Boyles Street, Frederick, Maryland 21702-1201, USA. Phone: 301.846.7456; Email: stcroixb@mail.nih.gov. 
S. Saha's present address is: Bristol-Myers Squibb, Princeton, New Jersey, USA.

XMZ's present address is: Kyn Therapeutics, Cambridge, Massachusetts, USA.
EZ's present address is: Janssen Pharmaceutical Companies, J\&J, Spring House, Pennsylvania, USA.

DSD's present address is: Center for Antibody Therapeutics, University of Pittsburgh Medical School, Pittsburgh, Pennsylvania, USA.
1. Beck A, Goetsch L, Dumontet C, Corvaïa N. Strategies and challenges for the next generation of antibody-drug conjugates. Nat Rev Drug Discov. 2017;16(5):315-337.

2. Kim EG, Kim KM. Strategies and Advancement in Antibody-Drug Conjugate Optimization for Targeted Cancer Therapeutics. Biomol Ther (Seoul). 2015;23(6):493-509.

3. Elenbaas B, Weinberg RA. Heterotypic signaling between epithelial tumor cells and fibroblasts in carcinoma formation. Exp Cell Res. 2001;264(1):169-184.

4. Rak JW, St Croix BD, Kerbel RS. Consequences of angiogenesis for tumor progression, metastasis and cancer therapy. Anticancer Drugs. 1995;6(1):3-18.

5. Folkman J. Tumor angiogenesis: therapeutic implications. N EnglJ Med.1971;285(21):1182-1186.

6. Dvorak HF. Tumors: wounds that do not heal. Similarities between tumor stroma generation and wound healing. N Engl JMed. 1986;315(26):1650-1659.

7. Schürch W, Seemayer TA, Lagacé R. Stromal myofibroblasts in primary invasive and metastatic carcinomas. A combined immunological, light and electron microscopic study. Virchows Arch A Pathol Anat Histol. 1981;391(2):125-139.

8. Abdollahi A, Folkman J. Evading tumor evasion: current concepts and perspectives of antiangiogenic cancer therapy. Drug Resist Updat. 2010;13(1-2):16-28.

9. Santos AM, Jung J, Aziz N, Kissil JL, Puré E. Targeting fibroblast activation protein inhibits tumor stromagenesis and growth in mice. JClin Invest. 2009;119(12):3613-3625.

10. Hwang RF, et al. Cancer-associated stromal fibroblasts promote pancreatic tumor progression. Cancer Res. 2008;68(3):918-926.

11. Orimo A, et al. Stromal fibroblasts present in invasive human breast carcinomas promote tumor growth and angiogenesis through elevated SDF-1/ CXCL12 secretion. Cell. 2005;121(3):335-348.

12. Olumi AF, Grossfeld GD, Hayward SW, Carroll PR, Tlsty TD, Cunha GR. Carcinoma-associated fibroblasts direct tumor progression of initiated human prostatic epithelium. Cancer Res. 1999;59(19):5002-5011.

13. Kalluri R. The biology and function of fibroblasts in cancer. Nat Rev Cancer. 2016;16(9):582-598.

14. Kraman M, et al. Suppression of antitumor immunity by stromal cells expressing fibroblast activation protein-alpha. Science. 2010;330(6005):827-830.

15. Brennen WN, Isaacs JT, Denmeade SR. Rationale behind targeting fibroblast activation proteinexpressing carcinoma-associated fibroblasts as a novel chemotherapeutic strategy. Mol Cancer Ther. 2012;11(2):257-266.

16. Roberts EW, et al. Depletion of stromal cells expressing fibroblast activation protein- $\alpha$ from skeletal muscle and bone marrow results in cachexia and anemia. JExp Med. 2013;210(6):1137-1151.

17. Tran E, et al. Immune targeting of fibroblast activation protein triggers recognition of multipotent bone marrow stromal cells and cachexia. JExp Med. 2013;210(6):1125-1135.

18. Oh P, et al. Subtractive proteomic mapping of the endothelial surface in lung and solid tumours for tissue-specific therapy. Nature. 2004;429(6992):629-635.

19. St Croix B, et al. Genes expressed in human tumor endothelium. Science. 2000;289(5482):1197-1202.

20. Seaman S, Stevens J, Yang MY, Logsdon D, Graff-Cherry C, St Croix B. Genes that distinguish physiological and pathological angiogenesis. Cancer Cell. 2007;11(6):539-554.

21. Nanda A, et al. TEM8 interacts with the cleaved C5 domain of collagen alpha 3(VI). Cancer Res. 2004;64(3):817-820.

22. Bagley RG, et al. Human mesenchymal stem cells from bone marrow express tumor endothelial and stromal markers. Int J Oncol. 2009;34(3):619-627.

23. Chaudhary A, et al. TEM8/ANTXR1 blockade inhibits pathological angiogenesis and potentiates tumoricidal responses against multiple cancer types. Cancer Cell. 2012;21(2):212-226.

24. Gutwein LG, Al-Quran SZ, Fernando S, Fletcher BS, Copeland EM, Grobmyer SR. Tumor endothelial marker 8 expression in triple-negative breast cancer. Anticancer Res. 2011;31(10):3417-3422.

25. Chaudhary A, St Croix B. Selective blockade of tumor angiogenesis. Cell Cycle. 2012;11(12):2253-2259.

26. Werner E, Kowalczyk AP, Faundez V. Anthrax toxin receptor 1/tumor endothelium marker 8 mediates cell spreading by coupling extracellular ligands to the actin cytoskeleton. J Biol Chem. 2006;281(32):23227-23236.

27. Reeves CV, et al. Anthrax toxin receptor 2 functions in ECM homeostasis of the murine reproductive tract and promotes MMP activity. PLoS One. 2012;7(4):e34862.

28. Hotchkiss KA, Basile CM, Spring SC, Bonuccelli G, Lisanti MP, Terman BI. TEM8 expression stimulates endothelial cell adhesion and migration by regulating cell-matrix interactions on collagen. Exp Cell Res. 2005;305(1):133-144.

29. Cullen M, et al. Host-derived tumor endothelial marker 8 promotes the growth of melanoma. Cancer Res. 2009;69(15):6021-6026.

30. Carson-Walter EB, Watkins DN, Nanda A, Vogelstein B, Kinzler KW, St Croix B. Cell surface tumor endothelial markers are conserved in mice and humans. Cancer Res. 2001;61(18):6649-6655.

31. Cheang MC, et al. Immunohistochemical detection using the new rabbit monoclonal antibody SP1 of estrogen receptor in breast cancer is superior to mouse monoclonal antibody 1D5 in predicting survival. JClin Oncol. 2006;24(36):5637-5644.

32. Bagley RG, et al. Tumor endothelial marker 7 (TEM-7): a novel target for antiangiogenic therapy. Microvasc Res. 2011;82(3):253-262.

33. Yang MY, et al. The cell surface structure of tumor endothelial marker 8 (TEM8) is regulated by the actin cytoskeleton. Biochim Biophys Acta. 2011;1813(1):39-49.

34. Maderna A, Leverett CA. Recent advances in the development of new auristatins: structural modifications and application in antibody drug conjugates. Mol Pharm. 2015;12(6):1798-1812.

35. Hamblett KJ, et al. Effects of drug loading on the antitumor activity of a monoclonal antibody drug conjugate. Clin Cancer Res. 2004;10(20):7063-7070.

36. Doronina SO, et al. Development of potent monoclonal antibody auristatin conjugates for cancer therapy. Nat Biotechnol. 2003;21(7):778-784.

37. Francisco JA, et al. cAC10-vcMMAE, an antiCD30-monomethyl auristatin $E$ conjugate with potent and selective antitumor activity. Blood. 2003;102(4):1458-1465.

38. Dorywalska M, et al. Molecular basis of valinecitrulline-PABC linker instability in site-specific ADCs and its mitigation by linker design. $\mathrm{Mol}$ Cancer Ther. 2016;15(5):958-970.

39. Seaman S, et al. Eradication of tumors through simultaneous ablation of CD276/B7-H3-positive tumor cells and tumor vasculature. Cancer Cell. 2017;31(4):501-515.e8.

40. Yu SF, et al. A novel anti-CD22 anthracycline-based antibody-drug conjugate (ADC) that overcomes resistance to auristatin-based ADCs. Clin Cancer Res. 2015;21(14):3298-3306.

41. Chen R, et al. CD30 downregulation, MMAE resistance, and MDR1 upregulation are all associated with resistance to brentuximab vedotin. $\mathrm{Mol}$ Cancer Ther. 2015;14(6):1376-1384.

42. Szakács G, et al. Predicting drug sensitivity and resistance: profiling $\mathrm{ABC}$ transporter genes in cancer cells. Cancer Cell. 2004;6(2):129-137.

43. Hamilton TC, et al. Characterization of a human ovarian carcinoma cell line (NIH:OVCAR-3) with androgen and estrogen receptors. Cancer Res. 1983;43(11):5379-5389.

44. Cailleau R, Young R, Olivé M, Reeves WJ. Breast tumor cell lines from pleural effusions. J Natl Cancer Inst. 1974;53(3):661-674.

45. Dillon RL, et al. Trastuzumab-deBouganin Conjugate Overcomes Multiple Mechanisms of T-DM1 Drug Resistance. J Immunother. 2016;39(3):117-126

46. Shefet-Carasso L, Benhar I. Antibody-targeted drugs and drug resistance--challenges and solutions. Drug Resist Updat. 2015;18:36-46.

47. Tumey LN, et al. Site Selection: a Case Study in the Identification of Optimal Cysteine Engineered Antibody Drug Conjugates. AAPS J. 2017;19(4):1123-1135. 
48. Vollmar BS, et al. Attachment Site Cysteine Thiol pKa is a Key driver for site-dependent stability of THIOMAB antibody-drug conjugates. Bioconjug Chem. 2017;28(10):2538-2548.

49. Burke PJ, et al. Optimization of a PEGylated glucuronide-monomethylauristatin E linker for antibody-drug conjugates. Mol Cancer Ther. 2017;16(1):116-123.

50. Gerber HP, Sapra P, Loganzo F, May C. Combining antibody-drug conjugates and immunemediated cancer therapy: What to expect? Biochem Pharmacol. 2016;102:1-6.

51. Müller P, et al. Microtubule-depolymerizing agents used in antibody-drug conjugates induce antitumor immunity by stimulation of dendritic cells. Cancer Immunol Res. 2014;2(8):741-755.

52. Tanaka H, Matsushima H, Nishibu A, Clausen BE, Takashima A. Dual therapeutic efficacy of vinblastine as a unique chemotherapeutic agent capable of inducing dendritic cell maturation. Cancer Res. 2009;69(17):6987-6994.

53. Tanaka H, Matsushima H, Mizumoto N, Takashima A. Classification of chemotherapeutic agents based on their differential in vitro effects on dendritic cells. Cancer Res. 2009;69(17):6978-6986.

54. Mizumoto N, Gao J, Matsushima H, Ogawa Y, Tanaka H, Takashima A. Discovery of novel immunostimulants by dendritic-cell-based functional screening. Blood. 2005;106(9):3082-3089.

55. Xu L, et al. COX-2 inhibition potentiates antiangiogenic cancer therapy and prevents metastasis in preclinical models. Sci Transl Med. 2014;6(242):242ra84.

56. Hingorani SR, et al. Trp53R172H and KrasG12D cooperate to promote chromosomal instability and widely metastatic pancreatic ductal adenocarcinoma in mice. Cancer Cell. 2005;7(5):469-483.

57. Zhu Z, Dimitrov DS. Construction of a large naïve human phage-displayed Fab library through one-step cloning. Methods Mol Biol. 2009;525:129-142, xv.

58. Jat PS, et al. Direct derivation of conditionally immortal cell lines from an $\mathrm{H}-2 \mathrm{~Kb}-\mathrm{ts} \mathrm{A} 58$ transgenic mouse. Proc Natl Acad Sci U S A. 1991;88(12):5096-5100. 